\title{
The smallest nontrivial snarks of oddness 4
}

\author{
Jan Goedgebeur* \\ Department of Applied Mathematics, Computer Science and Statistics \\ Ghent University \\ 9000 Ghent, Belgium \\ Computer Science Department \\ University of Mons \\ 7000 Mons, Belgium \\ jan.goedgebeur@ugent.be \\ Edita Máčajová ${ }^{\dagger}$ \\ Department of Computer Science \\ Comenius University \\ 84248 Bratislava, Slovakia \\ macajova@dcs.fmph.uniba.sk \\ Martin Škoviera ${ }^{\dagger}$ \\ Department of Computer Science \\ Comenius University \\ 84248 Bratislava, Slovakia \\ skoviera@dcs.fmph.uniba.sk
}

Submitted: XX; Accepted: XX; Published: XX

(C) The authors. Released under the CC BY-ND license (International 4.0).

\begin{abstract}
The oddness of a cubic graph is the smallest number of odd circuits in a 2-factor of the graph. This invariant is widely considered to be one of the most important measures of uncolourability of cubic graphs and as such has been repeatedly reoccurring in numerous investigations of problems and conjectures surrounding snarks (connected cubic graphs admitting no proper 3-edge-colouring). In [Ars Math. Contemp. 16 (2019), 277-298] we have proved that the smallest number of vertices of a snark with cyclic connectivity 4 and oddness 4 is 44 . We now show that there
\end{abstract}

${ }^{*}$ Supported by a Postdoctoral Fellowship of the Research Foundation Flanders (FWO).

${ }^{\dagger}$ Partially supported by VEGA 1/0876/16, VEGA 1/0813/18, and by APVV-15-0220. 
are exactly 31 such snarks, all of them having girth 5 . These snarks are built up from subgraphs of the Petersen graph and a small number of additional vertices. Depending on their structure they fall into six classes, each class giving rise to an infinite family of snarks with oddness at least 4 with increasing order. We explain the reasons why these snarks have oddness 4 and prove that the 31 snarks form the complete set of snarks with cyclic connectivity 4 and oddness 4 on 44 vertices. The proof is a combination of a purely theoretical approach with extensive computations performed by a computer.

Mathematics Subject Classifications: 05C15, 05C21, 05C30, 05C40, 05C75, $68 \mathrm{R} 10$

\section{Introduction}

This paper is a sequel of [8] where we have proved that the smallest number of vertices of a snark - a connected cubic graph whose edges cannot be properly coloured with three colours - which has cyclic connectivity 4 and oddness at least 4 is 44 . The purpose of the present paper is to show that there are precisely 31 such snarks, all of them having oddness exactly 4 , resistance 3 , and girth 5 . Together with [8], this paper provides a partial answer to the following question posed in [4, Problem 2], leaving open the existence of cyclically 5 -edge-connected snarks of oddness at least 4 on fewer than 44 vertices:

Problem [4]. Which is the smallest snark (with cyclic connectivity $\geqslant 4$ and girth $\geqslant 5$ ) of oddness strictly greater than 2 ?

The oddness of a bridgeless cubic graph $G$ is the smallest number of odd circuits in a 2-factor of $G$, and the resistance of $G$ is the smallest number of vertices (or edges) of $G$ whose removal yields a 3-edge-colourable graph. Both invariants are important measures of uncolourability of cubic graphs and have been investigated by numerous authors $[1,5,11,12,13,15,25]$. One of the reasons why these invariants have recently received so much attention resides in the fact that snarks with large resistance or oddness may provide potential counterexamples to several profound conjectures such as the cycle double cover conjecture, the 5 -flow conjecture, and others $[11,13,14]$.

The set $\mathcal{M}$ of all 31 snarks of order 44 , cyclic connectivity 4 , and oddness 4 has been constructed with the help of a computer. A detailed description of its members appears in Section 3 where we also give a computer-free proof that each of them has oddness at least 4 . The equality can be established easily by specifying a 2-factor containing four odd circuits, which can be checked directly.

The snarks constituting the set $\mathcal{M}$ fall into six classes depending on their structure. A remarkable feature of the set is that all of them are built up from subgraphs of the Petersen graph and a small number of additional vertices. The verification that $\mathcal{M}$ is a complete set of snarks of order 44 , cyclic connectivity 4 , and oddness at least 4 combines purely mathematical considerations with extensive computations; the proof can be found in Section 4. Its mathematical part is essentially a mixture of edge-colouring and cyclic connectivity arguments. In the computational part we perform an operation that takes 
two cyclically 4-edge-connected snarks $G_{1}$ and $G_{2}$ of order at most 36 and creates from them - in all possible ways - a cubic graph by either removing two adjacent vertices or two nonadjacent edges, and connecting the resulting 2 -valent vertices in $G_{1}$ with those in $G_{2}$. In total, more than $2 \times 10^{13}$ graphs have been constructed and checked for oddness 4 . The entire computational effort for this project amounts to $25 \mathrm{CPU}$ years.

In Section 5 we analyse a sample of 887152 cyclically 4-edge-connected snarks of oddness 4 whose orders range from 46 to 52 , as well as 872 snarks of oddness 4 with lower connectivity constructed in [9]. We evaluate various invariants for them such as resistance, perfect matching index, circumference, and others. The purpose of this investigation is to provide grounds for possible prediction of certain properties that snarks with higher oddness might have in general.

We conclude this paper with several open problems. At the end we append the adjacency lists of all 31 snarks constituting the set $\mathcal{M}$.

\section{Preliminaries}

This section collects the most basic definitions and notation needed for understanding the present paper. For a more detailed introduction to the topic we refer the reader to our preceding paper [8].

2.1. Graphs and multipoles. All graphs in this paper are finite and for the most part simple. However, for the sake of completeness we have to permit graphs containing multiple edges or loops, although these features will be usually excluded by the imposed connectivity or colouring restrictions. For a graph $G$ and a subgraph $H \subseteq G$ we let $|G|$ denote the number of vertices of $G$, and $G[H]$ the subgraph of $G$ induced by the vertex set of $H$.

Throughout this paper we use multipoles as a convenient tool for constructing graphs. Every edge of a multipole has two ends and each end can, but need not, be incident with a vertex. An edge which has one end incident with a vertex and the other not is called a dangling edge, and if neither end of an edge is incident with a vertex, it is called an isolated edge. An end of an edge that is not incident with a vertex is called a semiedge. A multipole with $k$ semiedges is called a $k$-pole. Two semiedges $s$ and $t$ of a multipole can be joined to produce an edge $s * t$ connecting the end-vertices of the corresponding dangling edges. Given two $k$-poles $M$ and $N$ with semiedges $s_{1}, \ldots, s_{k}$ and $t_{1}, \ldots, t_{k}$, respectively, we define their complete junction $M * N$ to be the graph obtained by performing the junctions $s_{i} * t_{i}$ for each $i \in\{1, \ldots, k\}$. A partial junction is defined in a similar way except that a proper subset of semiedges of $M$ is joined to semiedges of $N$. Partial junctions can be used to construct larger multipoles from smaller ones. In either case, whenever a junction of two multipoles is to be performed, we assume that their semiedges are assigned a fixed linear order.

Semiedges in multipoles are often grouped into pairwise disjoint sets, called connectors. The size of a connector is the number of its semiedges. A connector of size $n$ is often referred to as an $n$-connector. An $\left(n_{1}, n_{2}, \ldots, n_{k}\right)$-pole is a multipole with $n_{1}+n_{2}+\cdots+n_{k}$ 
semiedges which are distributed into $k$ connectors $S_{1}, S_{2}, \ldots, S_{k}$ such that the connector $S_{i}$ is of size $n_{i}$. A multipole with two connectors is also called a dipole.

Let $D^{\prime}\left(S_{1}^{\prime}, S_{2}^{\prime}\right)$ and $D^{\prime \prime}\left(S_{1}^{\prime \prime}, S_{2}^{\prime \prime}\right)$ be two dipoles with connectors $S_{1}^{\prime}, S_{2}^{\prime}$ and $S_{1}^{\prime \prime}, S_{2}^{\prime \prime}$, respectively. If $\left|S_{1}^{\prime}\right|=\left|S_{2}\right|$, and each of these two connectors is endowed with a linear order, we can construct a new dipole

$$
D\left(S_{1}, S_{2}\right)=D^{\prime}\left(S_{1}^{\prime}, S_{2}^{\prime}\right) \circ D^{\prime \prime}\left(S_{1}^{\prime \prime}, S_{2}^{\prime \prime}\right)
$$

with connectors $S_{1}=S_{1}^{\prime}$ and $S_{2}=S_{2}^{\prime \prime}$ by performing the junctions of the semiedges from $S_{2}^{\prime}$ with those $S_{1}^{\prime \prime}$ with respect to the corresponding orderings. The resulting dipole is called the junction of $D^{\prime}\left(S_{1}^{\prime}, S_{2}^{\prime}\right)$ and $D^{\prime \prime}\left(S_{1}^{\prime \prime}, S_{2}^{\prime \prime}\right)$.

2.2. Cyclic connectivity. Let $G$ be a connected graph. An edge-cut of a graph $G$ is any set $S$ of edges of $G$ such that $G-S$ is disconnected. For example, if $H$ a proper subset of vertices or induced subgraph of $G$, then the set $\delta_{G}(H)$ of all edges with exactly one end in $H$ is an edge-cut in $G$. An edge-cut $S$ is said to be cycle-separating if at least two components of $G-S$ contain cycles. We say that a connected graph $G$ is cyclically $k$-edge-connected if no set of fewer than $k$ edges is cycle-separating in $G$. Let $\beta(G)=|E(G)|-|V(G)|+1$ denote the cycle rank of $G$. The cyclic connectivity of $G$, denoted by $\zeta(G)$, is the largest number $k \leqslant \beta(G)$ for which $G$ is cyclically $k$-connected (cf. [20, 22]). It is not difficult to see that $\zeta(G)=\beta(G)$ if and only if $G$ any two curcuits of $G$ have a vertex in common. For cubic graphs this can happen only when $G$ is the complete bipartite graph $K_{3,3}$, the complete graph $K_{4}$ on four vertices, or the graph consisting of two vertices and three parallel edges joining them.

For a cubic graph $G$ with $\zeta(G) \leqslant 3$, the value $\zeta(G)$ coincides with the usual vertexconnectivity and edge-connectivity of $G$, so cyclic connectivity provides a natural extension of the classical connectivity parameters for cubic graphs. Another useful observation is that the value of cyclic connectivity remains invariant under subdivisions and adjoining new vertices of degree 1. In particular, homeomorphic graphs have the same value of cyclic connectivity.

For edge-cuts that separate an acyclic component from the rest of the graph we have the following easy but useful observation.

Lemma 1. A connected acyclic $k$-pole has $k-2$ vertices.

2.3. Edge-colourings. A 3-edge-colouring of a graph $G$ is a mapping $\varphi: E(G) \rightarrow$ $\{1,2,3\}$ such that adjacent edges receive distinct colours; the same definition applies to multipoles. A graph or a multipole which admits a 3-edge-colouring will be called colourable, otherwise it will be called uncolourable. A 2-connected uncolourable cubic graph is called a snark. A snark is nontrivial if it is cyclically 4-edge-connected and has girth at least 5 .

In the study of colourings of cubic graphs it is often convenient to take the colours to be the non-zero elements of the group $\mathbb{Z}_{2} \times \mathbb{Z}_{2}$, because in this case 3 -edge-colourings correspond to nowhere-zero $\mathbb{Z}_{2} \times \mathbb{Z}_{2}$-flows. We identify the colours $(0,1),(1,0)$, and $(1,1)$ with 1,2 , and 3 , respectively. 
The following well known lemma - in fact, an immediate consequence of flow continuity - is a fundamental tool in the study of snarks.

Theorem 2. (Parity Lemma) Let $M$ be a k-pole endowed with a proper 3-edge-colouring with colours 1,2 , and 3 . If the set of all semiedges of $M$ contains $k_{i}$ edges of colour $i$ for $i \in\{1,2,3\}$, then

$$
k_{1} \equiv k_{2} \equiv k_{3} \equiv k \quad(\bmod 2) .
$$

In this paper we study snarks that are far from being 3-edge-colourable. Two measures of uncolourability are relevant for this paper. The oddness $\omega(G)$ of a bridgeless cubic graph $G$ is the smallest number of odd circuits in a 2-factor of $G$. The resistance $\rho(G)$ of a cubic graph $G$ is the smallest number of vertices of $G$ which have to be removed in order to obtain a colourable graph. Somewhat surprisingly, the required number of vertices to be deleted is the same as the number of edges that have to be deleted in order to get a 3-edge-colourable graph (see [24, Theorem 2.7]). In fact, in many cases it is more convenient to delete edges rather than vertices.

Obviously, if $G$ is colourable, then $\omega(G)=\rho(G)=0$. If $G$ is uncolourable, then both $\omega(G) \geqslant 2$ and $\rho(G) \geqslant 2$. Observe that for every bridgeless cubic graph $G$ we have $\rho(G) \leqslant \omega(G)$ since deleting one edge from each odd circuit in a 2-factor leaves a colourable graph. On the other hand, the Parity Lemma implies that $\rho(G)$ never equals 1 , which together with a standard Kempe chain recolouring argument yields that $\rho(G)=2$ if and only if $\omega(G)=2$ [24, Lemma 2.5]. The difference between $\omega(G)$ and $\rho(G)$ can be arbitrarily large in general [25], nevertheless, resistance can serve as a convenient lower bound for oddness because it is somewhat easier to handle.

By the Parity Lemma, every colouring of a 4-pole has one of the following types: 1111, 1122, 1212, and 1221 (for a precise definition of the type of a colouring see [8]). Observe that every colourable 4-pole admits at least two different types of colourings. Indeed, we can start with any colouring and switch the colours along an arbitrary Kempe chain to obtain a colouring of another type. Colourable 4-poles thus can have two, three, or four different types of colourings. Those attaining exactly two types are particularly important for the study of snarks; we call them colour-open 4-poles, as opposed to colourclosed multipoles discussed in more detail in [21].

There are two types of colour-open 4-poles. A 4-pole $M$ will be called isochromatic if its semiedges can be partitioned into two pairs such that in every colouring of $M$ the semiedges within each pair receive the same colour. A 4-pole $M$ will be called heterochromatic of its semiedges can be partitioned into two pairs such that in every colouring of $M$ the semiedges within each pair receive distinct colours. Typical examples of isochromatic and heterochromatic 4-poles are depicted in Figure 2 and Figure 3, respectively.

The adjectives "isochromatic" and "heterochromatic" can be similarly applied to graphs: a subgraph of a cubic graph will be called isochromatic if attaching a dangling edge to every 2-valent vertex produces an isochromatic 4-pole; a heterochromatic subgraph is defined similarly. 


\section{The 31 snarks}

Let $\mathcal{S}_{36}$ denote the set of all cyclically 4 -edge-connected snarks with at most 36 vertices; as mentioned in [8], the set $\mathcal{S}_{36}$ consists of 432105682 nonisomorphic graphs. For any two snarks $G_{1}$ and $G_{2}$ from $\mathcal{S}_{36}$ let us apply the following operation:

- From each $G_{i}$ form a 4-pole $M_{i}$ by either removing two adjacent vertices or two nonadjacent edges and by retaining the dangling edges.

- Construct a cubic graph $G$ by identifying the semiedges of $M_{1}$ with those of $M_{2}$ after possibly applying a permutation to the semiedges of $M_{1}$ or $M_{2}$.

The resulting graph $G$ will be called a 4 -join of $G_{1}$ and $G_{2}$. Define $\mathcal{M}$ to be the set all pairwise nonisomorphic cyclically 4 -edge-connected snarks of order 44 with oddness at least 4 that can be expressed as a 4 -join of two not necessarily distinct graphs from $\mathcal{S}_{36}$.

We have implemented a program which applies a 4-join in all possible ways to any two input graphs; see [8] for more details concerning the program. We have applied this program in all possible ways to every pair of graphs from $\mathcal{S}_{36}$ that lead to a graph on 44 vertices, and then tested which of the constructed graphs have oddness at least 4 . This computation took approximately $2 \mathrm{CPU}$ years on a cluster consisting of Intel Xeon E5-2660 CPU's at 2.60GHz and produced 31 snarks with oddness exactly 4.

Observation 1. The set $\mathcal{M}$ consists of exactly 31 snarks, each of them having oddness exactly 4 and girth 5 .

In the remainder of this section we describe the 31 snarks in detail and provide a computer-free proof that each of them has oddness at least 4. Their adjacency lists, displayed in the order as they were generated, can be found in Appendix. Graph number 28 is illustrated in Figure 1, two more of the 31 graphs are depicted in Figure 1 and Figure 4 in our previous paper [8] which represent graphs number 15 and 17, respectively.

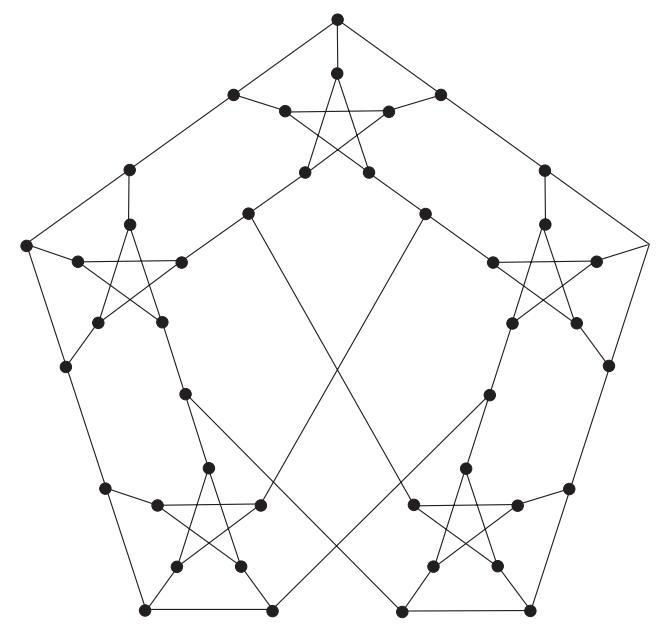

Figure 1: Graph number 28 of the 31 graphs in $\mathcal{M}$ 


\section{Building blocks}

The basic building blocks of all snarks constituting the set $\mathcal{M}$ are five multipoles $\mathbf{I}, \mathbf{H}_{1}$, $\mathbf{H}_{2}, \mathbf{T}$, and $\mathbf{N}$, described below, all of them arising from the Petersen graph by removing vertices or severing edges. Every individual member of $\mathcal{M}$ may have a small number of additional vertices not belonging to any of these subgraphs.

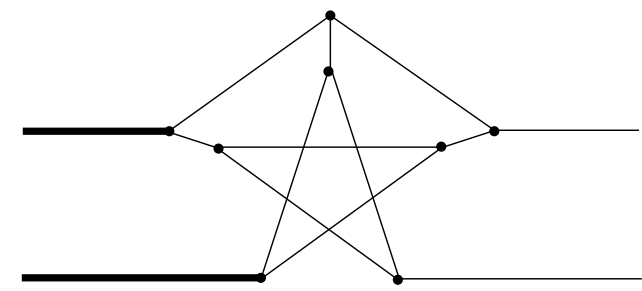

Figure 2: Building block I

1. Let $\mathbf{I}$ denote the $(2,2)$-pole arising from the Petersen graph by removing two adjacent vertices and grouping the semiedges formerly incident with the same vertex to the same connector; it is shown in Figure 2. Since every 3-edge-colouring of $\mathbf{I}$ assigns the edges in the same connector the same colour, $\mathbf{I}$ is an is an isochromatic 4-pole. It is the only isochromatic 4-pole on eight vertices and at the same time the smallest connected isochromatic 4-pole. In the symbolic representation of $\mathbf{I}$ we represent the edges in one of the connectors by bold lines, see Figure 6. This clearly determines the other connector as well. Due to the symmetry of the Petersen graph, the two connectors of $\mathbf{I}$ are interchangeable.

We emphasise that the connectors of $\mathbf{I}$, as well as those of the other four building blocks $\mathbf{H}_{1}, \mathbf{H}_{2}, \mathbf{T}$, and $\mathbf{N}$, are unordered. Different orderings are needed for the construction of the members of $\mathcal{M}$, a fact which partially explains a relatively large size of this set.
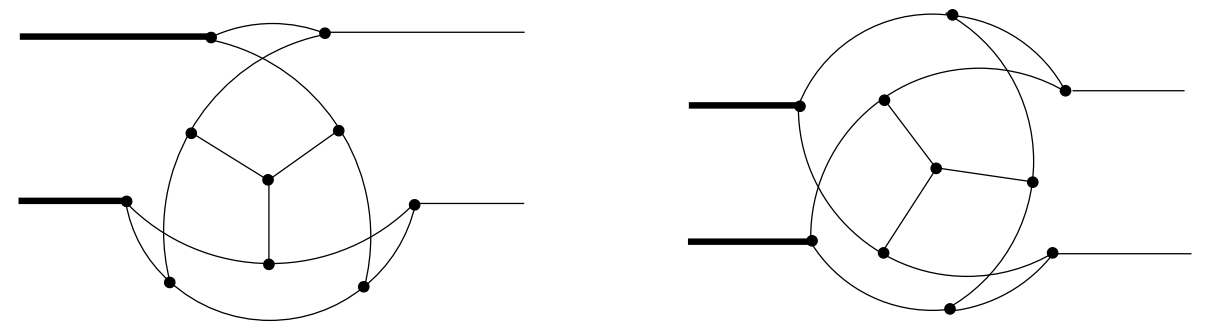

Figure 3: Building blocks $\mathbf{H}_{1}$ and $\mathbf{H}_{2}$

2. Let $\mathbf{H}$ denote a $(2,2)$-pole formed from the Petersen graph by severing two independent edges and grouping the semiedges arising from the same edge to the same connector. Every 3-edge-colouring of $\mathbf{H}$ assigns different colours to the dangling edges within the same connector, so $\mathbf{H}$ is a heterochromatic 4-pole. There are two ways how to select a pair of independent edges in the Petersen graph - either at 
distance 1 or at distance 2. Accordingly, there exist two nonisomorphic heterochromatic 4-poles on ten vertices, denoted by $\mathbf{H}_{1}$ and $\mathbf{H}_{2}$, respectively. Observe that there exists no bridgeless heterochromatic 4-pole with fewer vertices. The two heterochromatic 4-poles on ten vertices are displayed in Figure 3. In the symbolic representation of $\mathbf{H}$ we distinguish the two connectors of $\mathbf{H}$ again by using bold lines for one of the connectors, see Figure 6. Again, the connectors of both $\mathbf{H}_{1}$ and $\mathbf{H}_{2}$ are interchangeable.

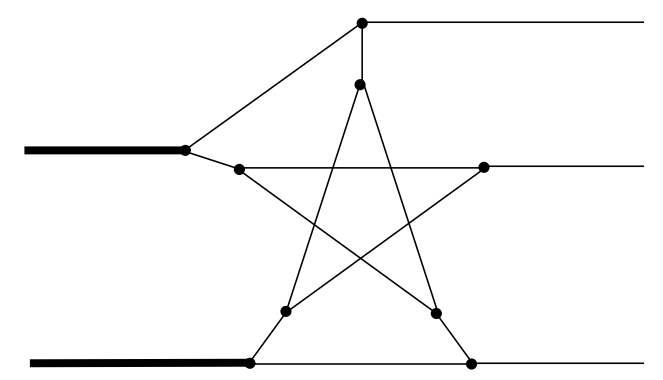

Figure 4: Building block T

3. Let $\mathbf{T}$ denote the (2,3)-pole obtained from the Petersen graph by removing an arbitrary vertex $v$ and severing an edge $e$ not incident with $v$; the semiedges formerly incident with $v$ are put to one connector and those arising by severing $e$ are put to the other connector. The resulting $(2,3)$-pole is shown in Figure 4. Given an arbitrary 3-edge-colouring of $\mathbf{T}$, the edges of the 2-connector receive two distinct colours $x$ and $y$, while the edges of the 3-connector receive the colours $x+y, z, z$, where $z$ is any colour from $\{1,2,3\}$. In the symbolic representation of $\mathbf{T}$ the edges of the 2-connector are drawn bold, see Figure 6.

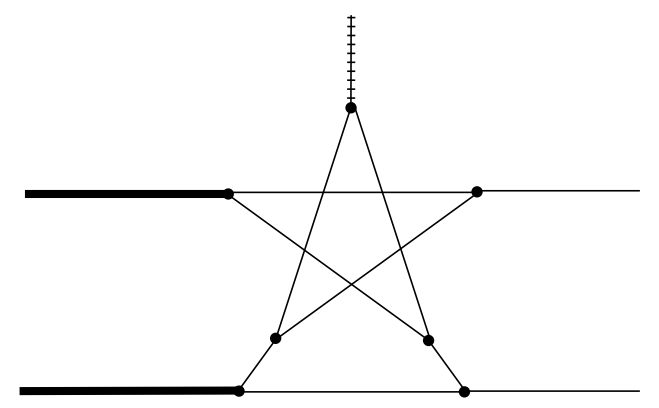

Figure 5: Building block $\mathbf{N}$

4. Let $\mathbf{N}$ denote the $(2,2,1)$-pole arising from the Petersen graph by removing a path of length 2. The two 2-connectors consist of the edges formerly incident with the same end-vertex of the path, the 1-connector gets the remaining edge. The resulting $(2,2,1)$-pole is shown in Figure 5 . The important property of $\mathbf{N}$ consists in the fact that every 3-edge-colouring of $\mathbf{N}$ assigns the edges of one of the 2-connectors two distinct colours $x$ and $y$ while the edges of the other 2-connector receive the 
same colour $z \in\{1,2,3\}$; the fifth edge of $\mathbf{N}$ is coloured $x+y$. In the symbolic representation of $\mathbf{N}$ the edges of one 2-connector are drawn bold and the 1-connector edge is drawn dashed, see Figure 6. As with $\mathbf{I}$ and $\mathbf{H}$ before, the two 2-connectors of $\mathbf{N}$ are interchangeable.
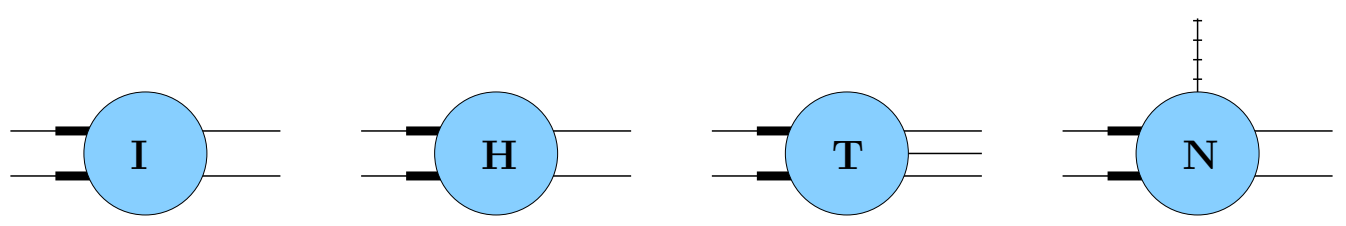

Figure 6: Basic building blocks

\section{Six classes}

We divide the 31 snarks of $\mathcal{M}$ into six classes depending on the number of disjoint copies of $\mathbf{I}, \mathbf{H}, \mathbf{T}$, and $\mathbf{N}$, and on the number of additional vertices in the graph. For example, by $2 \mathbf{H}+2 \mathbf{I}+\mathbf{N}+1$ we denote the set of all snarks from $\mathcal{M}$ that consist of two copies of $\mathbf{H}$, which need not be isomorphic, two copies of $\mathbf{I}$, one copy of $\mathbf{N}$, and one additional vertex. We do not distinguish between the two varieties $\mathbf{H}_{1}$ and $\mathbf{H}_{2}$ of $\mathbf{H}$ because both of them play the same structural role within the snark in question and their contribution to increasing the oddness is the same. The six classes of $\mathcal{M}$ are

$$
2 \mathbf{H}+3 \mathbf{I}, 2 \mathbf{H}+2 \mathbf{I}+\mathbf{N}+1, \mathbf{H}+4 \mathbf{I}+2, \mathbf{H}+3 \mathbf{I}+\mathbf{T}+1,5 \mathbf{I}+4, \text { and } 4 \mathbf{I}+\mathbf{T}+3 .
$$

In the rest of this section we describe each of the classes in detail and prove that every member $G \in \mathcal{M}$ has $\omega(G) \geqslant 4$. In our discussion we will be employing certain standard combinations of the multipoles $\mathbf{I}, \mathbf{H}$, and $\mathbf{T}$ defined by means of junctions. The order of semiedges in connectors is in all cases irrelevant. We define the $(2,2)$-poles $\mathbf{Z}_{1}=\mathbf{I} \circ \mathbf{H}$ and $\mathbf{Z}_{2}=\mathbf{I} \circ \mathbf{H} \circ \mathbf{I}$, the (2,3)-pole $\mathbf{Z}_{4}=\mathbf{I} \circ \mathbf{T}$, where the junction involves the 2-connector of $\mathbf{T}$, and the $(2,2,1)$-pole $\mathbf{Z}_{3}$ defined as follows: in the (2,2)-pole $\mathbf{I} \circ \mathbf{I}$ subdivide one of the edges between the two copies of $\mathbf{I}$ and subsequently attach a dangling edge to the new vertex of degree 2 ; the connectors of $\mathbf{Z}_{3}$ are defined in the obvious way. The multipoles $\mathbf{Z}_{1}, \mathbf{Z}_{2}, \mathbf{Z}_{3}$, and $\mathbf{Z}_{4}$ are illustrated in Figure 7 .

Lemma 3. The following statements hold true:

$$
\rho\left(\mathbf{Z}_{1}\right)=\rho\left(\mathbf{Z}_{3}\right)=\rho\left(\mathbf{Z}_{4}\right)=1 \text { and } \rho\left(\mathbf{Z}_{2}\right)=2 .
$$

Proof. Observe that $\mathbf{Z}_{1}$ is uncolourable because it is a junction of an isochromatic $(2,2)$ pole $\mathbf{I}$ with a heterochromatic $(2,2)$-pole $\mathbf{H}$. Hence, $\rho\left(\mathbf{Z}_{1}\right) \geqslant 1$. Similarly, every colouring of $\mathbf{T}$ assigns its 2-connector two distinct colours while $\mathbf{I}$ is isochromatic, so $\mathbf{Z}_{4}=\mathbf{I} \circ \mathbf{T}$ is uncolourable too, and therefore $\rho\left(\mathbf{Z}_{4}\right) \geqslant 1$ again. If the $(2,2,1)$-pole $\mathbf{Z}_{3}$ was colourable, then every 3 -edge-colouring of $\mathbf{Z}_{3}$ would assign the edges adjacent to the dangling edge of 


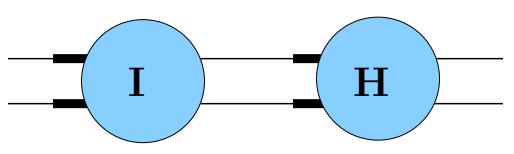

(a) dipole $\mathbf{Z}_{1}$

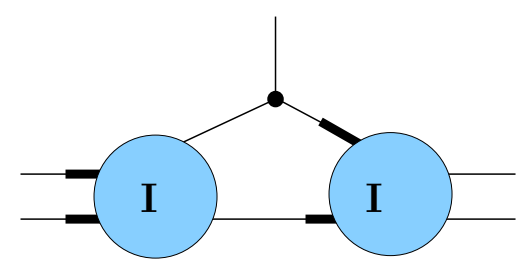

(c) Dipole $\mathbf{Z}_{3}$

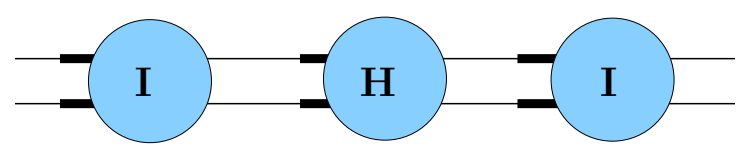

(b) Dipole $\mathbf{Z}_{2}$

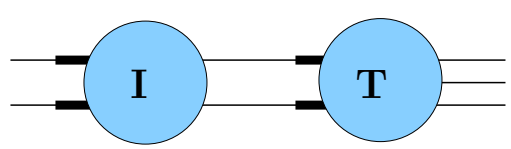

(d) Dipole $\mathbf{Z}_{4}$

Figure 7: Dipoles $\mathbf{Z}_{i}$

the 1-connector two distinct colours. At most one of these colours would match the colour of the edge connecting the two copies of $\mathbf{I}$ in $\mathbf{Z}_{3}$. Therefore, the isochromatic property of at least one copy of $\mathbf{I}$ in $\mathbf{Z}_{3}$ would always be violated. Hence, $\mathbf{Z}_{3}$ is uncolourable, and therefore $\rho\left(\mathbf{Z}_{3}\right) \geqslant 1$.

Finally we prove that $\rho\left(\mathbf{Z}_{2}\right) \geqslant 2$. Since $\mathbf{Z}_{1}$ is contained in $\mathbf{Z}_{2}$ and $\rho\left(\mathbf{Z}_{1}\right) \geqslant 1$, we infer that $\rho\left(\mathbf{Z}_{2}\right) \geqslant 1$ as well. In particular, the graph $K$ obtained from $\mathbf{Z}_{2}$ by identifying the semiedges within each connector is a snark. To prove that $\rho\left(\mathbf{Z}_{2}\right) \geqslant 2$ suppose to the contrary that $\rho\left(\mathbf{Z}_{2}\right)=1$, and let $v$ be a vertex such that $\mathbf{Z}_{2}-v$ is 3-edge-colourable. Clearly, $v$ cannot belong to a copy of $\mathbf{I}$ for otherwise $\mathbf{Z}_{2}-v$ would still contain a copy of $\mathbf{Z}_{1}$ and therefore would be uncolourable. Thus $v$ must belong to the copy of $\mathbf{H}$, and hence every 3-edge-colouring of $\mathbf{Z}_{2}-v$ must assign the same colour to both semiedges in any of the connectors. Now we can match the semiedges of each connector, thereby obtaining a 3-edge-colouring of $K-v$. Since $K$ is a snark, such a colouring does not exist. This contradiction proves that $\rho\left(\mathbf{Z}_{2}\right) \geqslant 2$.

To establish the required equalities for each particular multipole one has to display the corresponding colourings. Finding such colourings is straightforward, and therefore is left to the reader.

In our analysis of the 31 snarks we will often need to distinguish between different copies of the same basic building block $\mathbf{B} \in\{\mathbf{I}, \mathbf{H}, \mathbf{T}, \mathbf{N}\}$. For this purpose we will be using upper indices, for example $\mathbf{B}^{1}, \mathbf{B}^{2}$, etc.

\section{Class 1: $2 \mathrm{H}+3 \mathrm{I}$ (7 graphs)}

This class splits into two subclasses, Class 1a and Class 1b, depending on how the building blocks are connected between each other. Both subclasses are illustrated in Figure 8. Each graph $G$ from Class 1 contains a copy of $\mathbf{Z}_{1}$ and a copy of $\mathbf{Z}_{2}$. Since the copies of $\mathbf{Z}_{1}$ and $\mathbf{Z}_{2}$ are disjoint, Lemma 3 implies that $\rho(G) \geqslant 3$ and hence $\omega(G) \geqslant 4$.

Class 1a consists of graphs 15, 17, and 18, while Class 1b contains graphs 1, 4, 21, and 24 . 


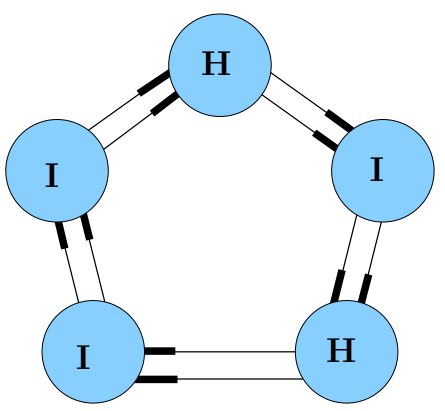

(a) Class 1a (3 graphs)

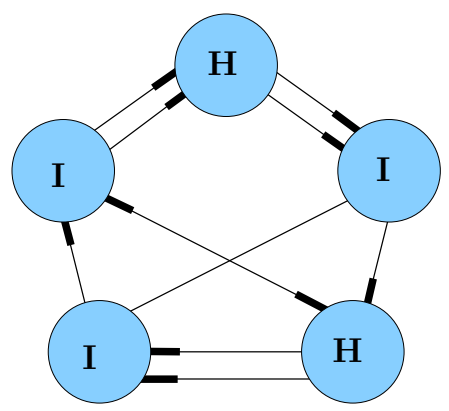

(b) Class 1b (4 graphs)

Figure 8: Class 1

\section{Class 2: $2 \mathrm{H}+2 \mathrm{I}+\mathrm{N}+1$ (4 graphs)}

The structure of graphs from Class 2 is illustrated in Figure 9. It can be seen that every graph $G$ from Class 2 contains two disjoint copies of $\mathbf{Z}_{1}$, therefore $\rho(G) \geqslant 2$. Note each of the induced subgraphs $\left[\mathbf{H}^{1} \cup \mathbf{N} \cup \mathbf{H}^{2}\right],\left[\mathbf{H}^{1} \cup \mathbf{I}^{1}\right],\left[\mathbf{H}^{2} \cup \mathbf{I}^{2}\right]$, and $\left[\mathbf{I}^{1} \cup \mathbf{I}^{2} \cup\{v\}\right]$ is uncolourable, the last of them being isomorphic to $\mathbf{Z}_{3}$. Thus if there exist two vertices $u$ and $w$ in $G$ such that $G-\{u, w\}$ is colourable, then either one of them lies in $\mathbf{H}^{1}$ and the other lies in $\mathbf{I}^{2}$, or one of them lies in $\mathbf{H}^{2}$ and the other lies in $\mathbf{I}^{1}$. In all other cases one of the mentioned uncolourable subgraphs will remain intact. Without loss of generality we may assume that $u$ belongs to $\mathbf{H}^{1}$ and $w$ belongs $\mathbf{I}^{2}$. Since $\mathbf{I}^{1}$ is isochromatic, the edges between $\mathbf{I}^{1}$ and $\mathbf{H}^{1}$ have the same colour. Because $\mathbf{H}^{2}$ is heterochromatic, the colours the edges between $\mathbf{H}^{2}$ and $\mathbf{N}$ are different. The inverting property of $\mathbf{N}$ ensures that the edges between $\mathbf{N}$ and $\mathbf{H}^{1}$ also have the same colour. If we now identify the dangling edges within each connector of $\mathbf{H}^{1}$, we obtain a colouring of the Petersen graph with one vertex removed. This contradiction proves that that $\rho(G)>2$, which means that $\omega(G) \geqslant 4$, as required.

Class 2 contains graphs 10, 11, 19, and 27.

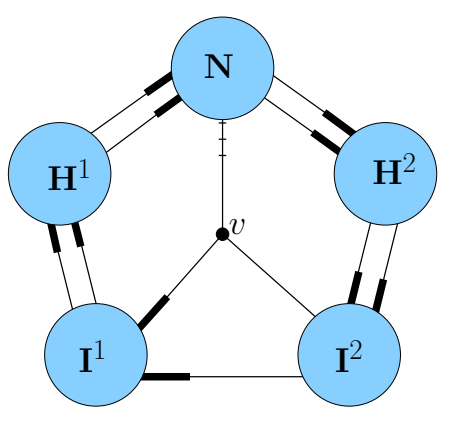

Figure 9: Class 2 (4 graphs) 


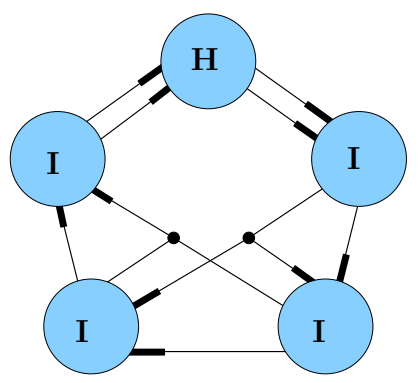

(a) Class 3a (2 graphs)

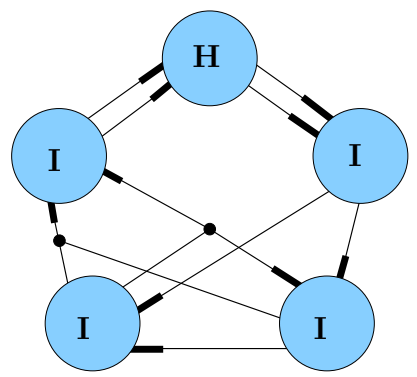

(b) Class 3b (2 graphs)

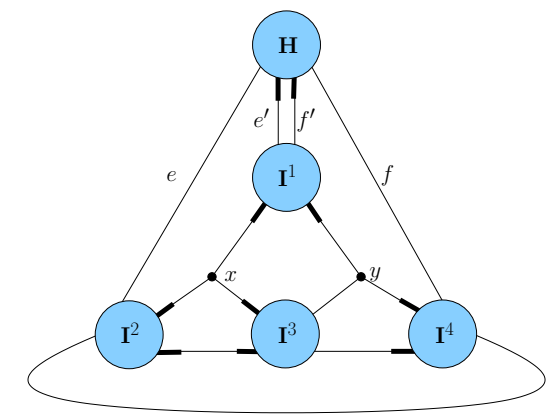

(c) Class 3c (2 graphs)

Figure 10: Class 3

\section{Class 3: $\mathrm{H}+4 \mathrm{I}+2$ (6 graphs)}

This class splits into three subclasses, 3a, 3b, and 3c. Their structure is represented in Figure 10. Every graph $G$ from Class 3 a and Class 3 b contains a copy of $\mathbf{Z}_{2}$ and a copy $\mathbf{Z}_{3}$, which are disjoint, therefore $\rho(G) \geqslant 3$ and $\omega(G) \geqslant 4$. Class 3c is somewhat different and requires a separate argument. Consider an arbitrary graph $G$ graph from Class 3c. Since $G$ contains a copy of $\mathbf{Z}_{1}$, we have $\rho(G) \geqslant 2$. Note that each of the subgraphs $\left[\mathbf{H} \cup \mathbf{I}^{1}\right]$, $\left[\mathbf{I}^{2} \cup \mathbf{I}^{3} \cup\{x\}\right]$, and $\left[\mathbf{I}^{3} \cup \mathbf{I}^{4} \cup\{y\}\right]$ has resistance 1 . Thus if there exist two vertices $u$ and $w$ in $G$ such that $G-\{u, w\}$ is 3-edge-colourable, then one of them, say $u$, lies in $\left[\mathbf{H} \cup \mathbf{I}^{1}\right]$ and the other belongs to $\mathbf{I}^{3}$. Since $\mathbf{I}^{2}$ and $\mathbf{I}^{4}$ remain intact in $G-\{u, w\}$, the isochromatic property of $\mathbf{I}$ implies that the edge $e$ connecting $\mathbf{I}^{2}$ to $\mathbf{H}$ has the same colour as the edge $f$ connecting $\mathbf{I}^{4}$ to $\mathbf{H}$. Since $\mathbf{H}$ is heterochromatic, we conclude that $u$ lies in $\mathbf{H}$. As a consequence, $\mathbf{I}^{1}$ remains intact in $G-\{u, w\}$ too, which means that the two edges $e^{\prime}$ and $f^{\prime}$ joining $\mathbf{H}$ to $\mathbf{I}^{1}$ are equally coloured as well. But then the induced colouring of $\mathbf{H}$ yields a 3-edge-colouring of the Petersen graph with a single vertex removed. This contradiction proves that $\rho(G)>2$ and $\omega(G) \geqslant 4$.

Class 3a contains graphs 5 and 6, Class 3b contains graphs 7 and 23, and Class 3c contains graphs 9 and 26 . 


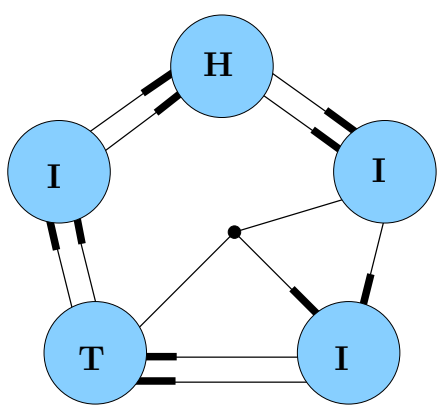

(a) Class 4a (4 graphs)

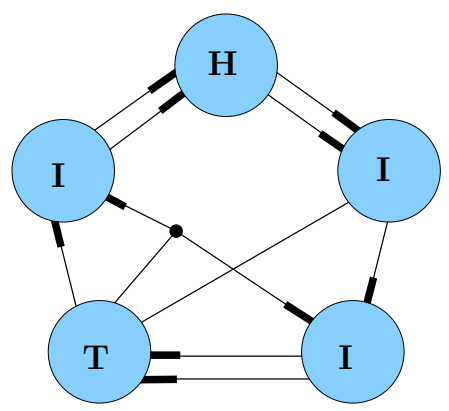

(b) Class 4b (6 graphs)

Figure 11: Class 4

\section{Class 4: $\mathrm{H}+3 \mathrm{I}+\mathrm{T}+1$ (10 graphs)}

Class 4 has two subclasses $4 \mathrm{a}$ and $4 \mathrm{~b}$, both shown in Figure 11. Every graph $G$ from Class 4 contains disjoint copies of $\mathbf{Z}_{2}$ and $\mathbf{Z}_{4}$, so $\rho(G) \geqslant 3$ and $\omega \geqslant 4$.

Class 4 a consists of graphs 2, 3, 13, and 14, and Class 4b contains graphs 8, 12, 16, 20, 22 , and 25 .

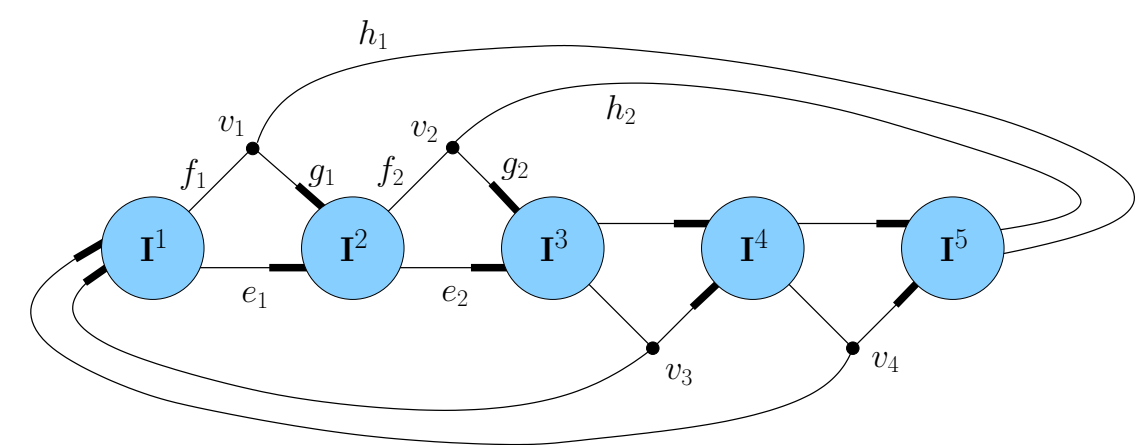

(a) Class 5a (1 graph)

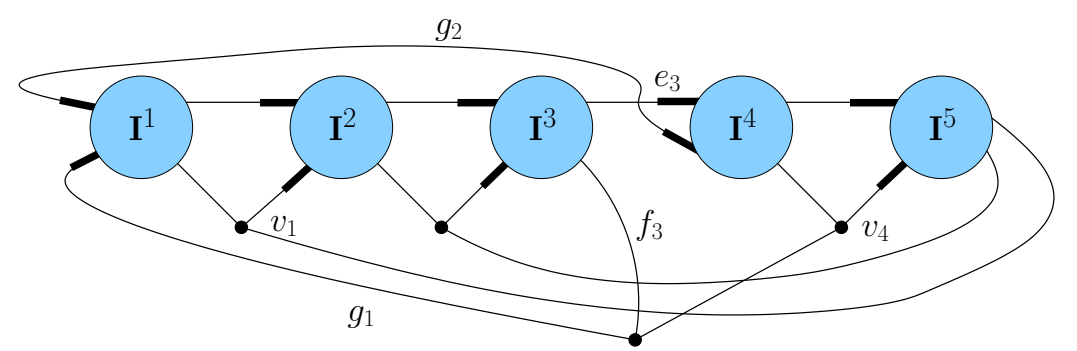

(b) Class 5b (1 graph)

Figure 12: Class 5 


\section{Class 5: $5 \mathrm{I}+4$ (2 graphs)}

Class 5 has two subclasses Class 5a and Class 5b; they are represented in Figure 12. Observe that every member of Class 5 contains a 5 -pole isomorphic to $\mathbf{Z}_{3}$, namely $\left[\mathbf{I}^{1} \cup\right.$ $\mathbf{I}^{2} \cup\left\{v_{1}\right\}$ ], whose resistance is 1 by Lemma 3. Hence, every member of Class 5 is indeed a snark.

Now, let us consider a graph $G$ from Class 5 a. To prove that $\omega(G) \geqslant 4$ suppose the contrary. Then $\omega(G)=\rho(G)=2$, which means that there exist vertices $u$ and $w$ in $G$ such that $G-\{u, w\}$ is colourable. Since each of the 5-poles $\left[\mathbf{I}^{k} \cup \mathbf{I}^{k+1} \cup\left\{v_{k}\right\}\right]$, with $k \in\{1,2,3,4\}$, is isomorphic to $\mathbf{Z}_{3}$, and $\rho\left(\mathbf{Z}_{3}\right)=1$, none of them survives in $G-\{u, w\}$. It follows that one of $u$ and $w$ lies in $\mathbf{I}^{2}$ and the other one lies in $\mathbf{I}^{4}$. Let $\varphi$ be a 3 edge-colouring of $G-\{u, w\}$. Without loss of generality we may assume that $\varphi\left(f_{1}\right)=1$, $\varphi\left(g_{1}\right)=2$ and $\varphi\left(h_{1}\right)=3$. Since $\mathbf{I}^{1}$ remains intact in $G-\{u, w\}$, we have $\varphi\left(e_{1}\right)=1$. Similarly, since $\mathbf{I}^{5}$ is intact, we have $\varphi\left(h_{2}\right)=\varphi\left(h_{1}\right)=3$. Consider the colours of $e_{2}$ and $f_{2}$. Since $\mathbf{I}^{3}$ remains intact $G-\{u, w\}$, we deduce that $\varphi\left(e_{2}\right)=\varphi\left(g_{2}\right)$ and hence $\varphi\left(e_{2}\right) \neq \varphi\left(f_{2}\right)$. Furthermore, $\{1,2\}=\left\{\varphi\left(e_{1}\right), \varphi\left(g_{1}\right)\right\} \neq\left\{\varphi\left(e_{2}\right), \varphi\left(f_{2}\right)\right\}$ because otherwise the induced colouring of $\mathbf{I}^{2}$ would give rise to a 3-edge-colouring of the Petersen graph with one vertex removed, which is impossible. Therefore $\left\{\varphi\left(e_{2}\right), \varphi\left(f_{2}\right)\right\}=\{1,3\}$ or $\{2,3\}$, but since $\varphi\left(f_{2}\right) \neq \varphi\left(h_{2}\right)=3$ we conclude that $\varphi\left(e_{2}\right)=3$. However, the isochromatic property of $\mathbf{I}^{3}$ forces $\varphi\left(g_{2}\right)=\varphi\left(e_{2}\right)=3$, which is in conflict with the value $\varphi\left(h_{2}\right)=3$ because $g_{2}$ and $h_{2}$ are adjacent. Therefore $\rho(G) \geqslant 3$ and $\omega(G) \geqslant 4$.

Next, let us consider a graph $G$ from Class 5 b. We wish to prove that $\omega(G) \geqslant 4$. Suppose the contrary. Then $\rho(G)=2$, which again means that there exist vertices $u$ and $w$ in $G$ such that $G-\{u, w\}$ is 3-edge-colourable. In this case it is easy to see that one of the vertices belongs $\mathbf{I}^{2}$ and the other one belongs to $\mathbf{I}^{4} \cup \mathbf{I}^{5} \cup\left\{v_{4}\right\}$. Suppose that the vertex $w$ lies in $\mathbf{I}^{5} \cup\left\{v_{4}\right\}$ so that $\mathbf{I}^{4}$ remains intact. Take an arbitrary 3-edge-colouring $\varphi$ of $G-\{u, w\}$, and let $\varphi\left(f_{3}\right)=x$. Since $\mathbf{I}^{1}$ and $\mathbf{I}^{3}$ are also left intact in $G-\{u, w\}$, we deduce that $\varphi\left(e_{3}\right)=x, \varphi\left(g_{2}\right)=x$, and finally $\varphi\left(g_{1}\right)=x$. However, the edges $g_{1}$ and $e_{3}$ are adjacent, so this is impossible. Therefore $w \in \mathbf{I}^{4}$, which means that $\mathbf{I}^{5}$ is left intact. In this situation we can derive a contradiction in a similar way as for Class 5a. This proves that $\omega(G) \geqslant 4$ for every graph from Class 5 b.

Both Class 5a and Class 5b consist of a single graph each - graphs 28 and 30, respectively.

\section{Class 6: $4 \mathrm{I}+\mathrm{T}+3$ (2 graphs)}

The structure of Class 6 is displayed in Figure 13. Let us first observe that every member $G$ of Class 6 is uncolourable because it contains the subgraph $\left[\mathbf{I}^{1} \cup \mathbf{T}\right]$ isomorphic to $\mathbf{Z}_{4}$, whose resistance equals 1 by Lemma 3. It follows that $\rho(G) \geqslant 2$. To prove that $\omega(G) \geqslant 4$ suppose to the contrary that $\omega(G)=2=\rho(G)$. Since each of the subgraphs $\left[\mathbf{I}^{k} \cup \mathbf{I}^{k+1} \cup\left\{v_{k}\right\}\right]$, with $k \in\{2,3\}$, is also uncolourable, there exist vertices $u \in \mathbf{I}^{3}$ and $w \in\left[\mathbf{I}^{1} \cup \mathbf{T}\right]$ such that $G-\{u, w\}$ is 3-edge-colourable. Let $\varphi$ be one such colouring. Without loss of generality we may assume that $\varphi\left(e_{1}\right)=1, \varphi\left(e_{2}\right)=2$, and $\varphi\left(e_{3}\right)=3$. Since $\mathbf{I}^{2}$ and $\mathbf{I}^{4}$ are left intact, we have $\varphi\left(f_{1}\right)=2$ and $\varphi\left(f_{2}\right)=3$. Put together, the 


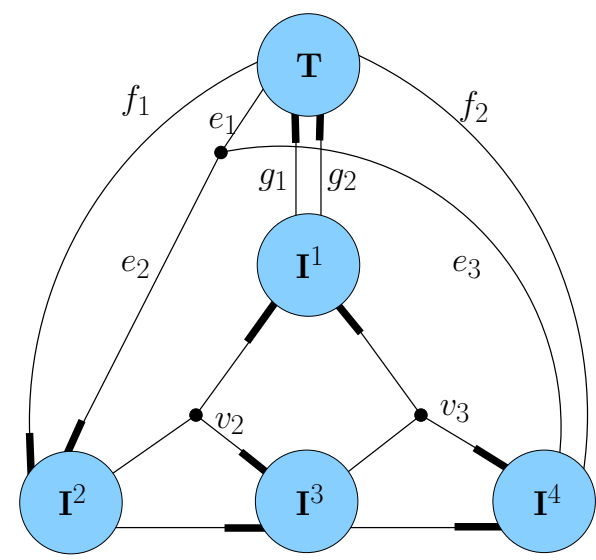

Figure 13: Class 6 (2 graphs)

edges of the 3-connector of $\mathbf{T}$ receive three distinct colours from the colouring $\varphi$. As previously mentioned, every 3 -edge-colouring of $\mathbf{T}$ forces a repeated colour in the 3 connector. Therefore $w$ must belong to $\mathbf{T}$. It follows that $\mathbf{I}^{1}$ remains intact and therefore $\varphi\left(g_{1}\right)=\varphi\left(g_{2}\right)$. But then the induced colouring of $\mathbf{T}$ yields a 3-edge-colouring of the Petersen graph with a single vertex removed. This contradiction proves that $\omega(G) \geqslant 4$.

Class 6 contains two nonisomorphic graphs -29 and 31 .

\section{Properties of graphs in $\mathcal{M}$}

We have determined the values of several invariants for the 31 snarks in $\mathcal{M}$. In most cases the computations were performed by a computer. The evaluated invariants can be divided into two groups. The first group is constituted by general invariants: namely, the order of the automorphism group, genus (minimum genus of an orientable surface upon which a given graph can be drawn without intersections), diameter, radius, and circumference (the maximal circuit length in a graph). The values of these invariants for individual members of $\mathcal{M}$ are summarised in Table 1. In particular, all members of $\mathcal{M}$ have circumference 41 , that is, $n-3$ where $n$ is the number of vertices. The values of the remaining invariants vary over the set $\mathcal{M}$. It is quite remarkable that the automorphism group of every graph in $\mathcal{M}$ is a 2 -group (or is trivial).

The second group of invariants comprises those which are of particular interest for snarks: perfect matching index $\pi$, resistance $\rho$, weak oddness $\omega^{\prime}$, and two invariants introduced in $[15,26,27]$ and denoted by $\mu_{3}$ and $\gamma_{2}$; see also [5] for a recent survey. The perfect matching index of a bridgeless cubic graph $G$, denoted by $\pi(G)$ (also known as excessive index and denoted by $\chi_{e}^{\prime}$ ), is the smallest number of perfect matchings that cover all the edges of $G[2,7]$. The weak oddness of a cubic graph $G$, denoted by $\omega^{\prime}(G)$, is the smallest number of components of odd order in an even factor of $G$; by an even factor we mean a spanning subgraph with all degrees even. Given a bridgeless cubic graph $G$, we define $\gamma_{2}(G)$ to be the smallest number of common edges that two perfect matchings of $G$ can have, and let $\mu_{3}(G)$ be the smallest number of edges of $G$ that are left uncovered by the union of any three perfect matchings of $G$. 


\begin{tabular}{|c|c|c|c|c|c|c|}
\hline Class & Graph number & $|\operatorname{Aut}(G)|$ & Genus & Diameter & Radius & Circumference \\
\hline $1 \mathrm{a}$ & 15 & 4 & 4 & 8 & 6 & 41 \\
\hline $1 \mathrm{a}$ & 17 & 64 & 5 & 8 & 7 & 41 \\
\hline $1 \mathrm{a}$ & 18 & 8 & 5 & 8 & 7 & 41 \\
\hline $1 \mathrm{~b}$ & 1 & 16 & 5 & 8 & 6 & 41 \\
\hline $1 \mathrm{~b}$ & 4 & 1 & 4 & 8 & 6 & 41 \\
\hline $1 \mathrm{~b}$ & 21 & 4 & 5 & 8 & 6 & 41 \\
\hline $1 \mathrm{~b}$ & 24 & 4 & 5 & 8 & 6 & 41 \\
\hline 2 & 10 & 2 & 4 & 8 & 5 & 41 \\
\hline 2 & 11 & 2 & 4 & 8 & 5 & 41 \\
\hline 2 & 19 & 16 & 5 & 8 & 5 & 41 \\
\hline 2 & 27 & 2 & 5 & 8 & 5 & 41 \\
\hline $3 a$ & 5 & 4 & 5 & 8 & 6 & 41 \\
\hline $3 \mathrm{a}$ & 6 & 1 & 4 & 8 & 6 & 41 \\
\hline $3 b$ & 7 & 2 & 5 & 8 & 6 & 41 \\
\hline $3 \mathrm{~b}$ & 23 & 8 & 5 & 8 & 6 & 41 \\
\hline $3 \mathrm{c}$ & 9 & 4 & 4 & 8 & 6 & 41 \\
\hline $3 \mathrm{c}$ & 26 & 1 & 4 & 8 & 6 & 41 \\
\hline $4 a$ & 2 & 8 & 5 & 8 & 7 & 41 \\
\hline $4 a$ & 3 & 4 & 5 & 8 & 7 & 41 \\
\hline $4 a$ & 13 & 2 & 4 & 8 & 6 & 41 \\
\hline $4 a$ & 14 & 1 & 4 & 8 & 6 & 41 \\
\hline $4 \mathrm{~b}$ & 8 & 1 & 4 & 8 & 6 & 41 \\
\hline $4 \mathrm{~b}$ & 12 & 1 & 4 & 8 & 6 & 41 \\
\hline $4 \mathrm{~b}$ & 16 & 4 & 5 & 8 & 6 & 41 \\
\hline $4 \mathrm{~b}$ & 20 & 1 & 5 & 8 & 6 & 41 \\
\hline $4 \mathrm{~b}$ & 22 & 4 & 5 & 8 & 6 & 41 \\
\hline $4 \mathrm{~b}$ & 25 & 4 & 5 & 8 & 6 & 41 \\
\hline $5 \mathrm{a}$ & 28 & 2 & 4 & 8 & 6 & 41 \\
\hline $5 \mathrm{~b}$ & 30 & 2 & 5 & 7 & 6 & 41 \\
\hline 6 & 29 & 2 & 5 & 7 & 6 & 41 \\
\hline 6 & 31 & 1 & 4 & 8 & 6 & 41 \\
\hline
\end{tabular}

Table 1: Basic invariant values for the 31 snarks of $\mathcal{M}$.

The perfect matching index $\pi(G)$ of $G$ is bounded below by 3 and equals 3 if and only if $G$ is 3-edge-colourable. It is believed, by a conjecture of Berge (see [23]), that $\pi(G) \leqslant 5$ for every bridgeless cubic graph $G$. This conjecture, if true, thus divides all snarks into two subclasses, those with perfect matching index equal to 4 , and those with perfect matching index 5 . We have determined that $\pi(G)=4$ for every $G \in \mathcal{M}$.

The remaining invariants, along with oddness, can be regarded as measures of un- 
colourability as they take value 0 on 3 -edge-colourable graphs, and positive values otherwise. Their comparison with oddness and resistance may therefore be very instructive.

First of all, using a computer we have determined that $\rho(G)=3$ for all snarks $G \in \mathcal{M}$. As regards weak oddness, it is easy to see that $\omega^{\prime}(G)$ is an even integer such that $\rho(G) \leqslant$ $\omega^{\prime}(G) \leqslant \omega(G)$. Furthermore, if $\omega(G)=4$, then necessarily $\omega^{\prime}(G)=4$ as well, because otherwise $\omega^{\prime}(G)=2$ would immediately yield $\rho(G)=2$ whence $\omega(G)=2$. In particular, for all snarks $G \in \mathcal{M}$ we have $\omega^{\prime}(G)=\omega(G)=4$. It is known, however, that in general both the difference $\omega(G)-\omega^{\prime}(G)$ and $\omega^{\prime}(G)-\rho(G)$ can be arbitrarily large (see $[1,17]$ ).

We finish this section by discussing the previously mentioned invariants $\gamma_{2}$ and $\mu_{3}$. In [27, Proposition 2.1] Steffen proved that $\omega(G) \leqslant 2 \gamma_{2}(G)$ for every bridgeless cubic graph $G$. Using a computer we have determined that $\gamma_{2}(G)=2$ for every $G \in \mathcal{M}$, which shows that every snark from $\mathcal{M}$ fulfils the upper bound on $\omega$ set by $\gamma_{2}$ with equality. Similarly, in [15, Corollary 2.4] Jin and Steffen proved that $\omega(G) \leqslant 2 \mu_{3}(G) / 3$ for every bridgeless cubic graph. Using a computer we have determined that all snarks $G \in \mathcal{M}$ have $\mu_{3}(G)=6$, which means that they again reach the upper bound on $\omega$ in terms of $\mu_{3}$ with equality. Snarks with the latter property have a very special structure of sets of edges left uncovered by three perfect matchings and therefore deserve special attention.

The values of perfect matching index, resistance, weak oddness, $\gamma_{2}$, and $\mu_{3}$ for the snarks of $\mathcal{M}$ are summarised in Table 2.

\begin{tabular}{rrrrr}
\hline$\pi$ & $\rho$ & $\omega^{\prime}$ & $\gamma_{2}$ & $\mu_{3}$ \\
\hline 4 & 3 & 4 & 2 & 6 \\
\hline
\end{tabular}

Table 2: Values of perfect matching index and unclourability measures for the snarks of $\mathcal{M}$

\section{Infinite families}

Each of the six classes described above gives rise to an infinite family of snarks with oddness at least 4 and cyclic connectivity 4 . It is sufficient to replace the basic building blocks $\mathbf{I}, \mathbf{H}, \mathbf{T}$, and $\mathbf{N}$ obtained from the Petersen graph with similar structures created from any cyclically 4-edge-connected snark. With a little additional care one can construct infinite families of snarks with increasing oddness.

\section{Completeness of $\mathcal{M}$}

In this section we prove that the set $\mathcal{M}$, constructed and analysed in Section 3 , is the complete set of pairwise nonisomorphic snarks with cyclic connectivity 4, oddness at least 4, and minimum order. Our point of departure is the following theorem proved in [8].

Theorem 4. The smallest number of vertices of a snark with cyclic connectivity 4 and oddness at least 4 is 44 . The girth of each such snark is at least 5. 
This result is a consequence of the following stronger and more detailed result from [8] which will be needed for the proof of the main result of this paper.

Theorem 5. Let $G$ be a snark with oddness at least 4, cyclic connectivity 4, and minimum number of vertices. Let $S$ be a cycle-separating 4-edge-cut in $G$ whose removal leaves components $G_{1}$ and $G_{2}$. Then, up to permutation of the index set $\{1,2\}$, exactly one of the following occurs.

(i) Both $G_{1}$ and $G_{2}$ are uncolourable, in which case each of them can be extended to a cyclically 4-edge-connected snark by adding two vertices.

(ii) $G_{1}$ is uncolourable and $G_{2}$ is heterochromatic, in which case $G_{1}$ can be extended to a cyclically 4-edge-connected snark by adding two vertices, and $G_{2}$ can be extended to a cyclically 4-edge-connected snark by adding two isolated edges.

(iii) $G_{1}$ is uncolourable and $G_{2}$ is isochromatic, in which case $G_{1}$ can be extended to a cyclically 4-edge-connected snark by adding two vertices, and $G_{2}$ can be extended to a cyclically 4-edge-connected snark by adding two vertices, except possibly $\zeta\left(G_{2}\right)=2$. In the latter case, $G_{2}$ is a partial junction of two colour-open 4-poles, which may be isochromatic or heterochromatic in any combination.

Here is our main result:

Theorem 6. The set $\mathcal{M}$ is the complete set of snarks with cyclic connectivity 4 and oddness at least 4 on 44 vertices.

Let $\mathcal{N}$ be the set of all snarks with cyclic connectivity 4 and oddness at least 4 on 44 vertices. To prove Theorem 6 it suffices to show that $\mathcal{N} \subseteq \mathcal{M}$. The general strategy of the proof is to show that every snark $G \in \mathcal{N}$ is a 4-join of two cyclically 4-edge-connected snarks of order at most 36. As soon as this is done, one can perform 4-joins in all possible ways that give rise to a cyclically 4-edge-connected snark of order 44, identify those whose oddness equals 4 , and check whether all of them belong to $\mathcal{M}$.

In order to apply this strategy we employ Theorem 5. It implies that we can split each $G \in \mathcal{N}$ into two subgraphs $G_{1}$ and $G_{2}$ each of which can be extended to cyclically a 4-edge-connected snark by adding at most two vertices. The difficult part of the proof arises when one of the subgraphs, namely $G_{1}$, has 36 vertices and $G_{2}$ is an isochromatic 4 -pole on 8 vertices (see (iii) of Theorem 5). Adding two adjacent vertices to $G_{1}$ is now useless because the list of all cyclically 4-edge-connected snarks is known only up to 36 vertices $[4,8]$. Instead, we show that it is possible to add two isolated edges to $G_{1}$ in such a way that a cyclically 4-edge-connected snark of order 36 is created. A detailed analysis that precedes this step is the core of the proof of Theorem 6, which now follows.

Proof. Let $G$ be a snark with cyclic connectivity 4 and oddness at least 4 on 44 vertices. We wish to prove that $G \in \mathcal{M}$. Suppose the contrary. In order to derive a contradiction we first establish four claims. 
Claim 1. Every cycle-separating 4-edge-cut of $G$ determines two components, one uncolourable on 36 and one isochromatic on 8 vertices. Both components are 2-edge-connected.

Proof of Claim 1. Let $S$ be an arbitrary cycle-separating 4-edge-cut in $G$ and let $G_{1}$ and $G_{2}$ be the components of $G-S$. Since $S$ is a minimum cycle-separating edge-cut, both $G_{1}$ and $G_{2}$ are easily seen to be 2-edge-connected. ¿From Theorem 5 we deduce that one of the components, say $G_{1}$, is uncolourable. If $G_{2}$ was either uncolourable or heterochromatic, then it would have at least 10 vertices and hence $G_{1}$ has at most 34 vertices. Using Theorem 5 again we could conclude that both $G_{1}$ and $G_{2}$ can be extended to snarks of order at most 36 , so $G$ would be a 4 -join of two snarks from $\mathcal{S}_{36}$ and therefore a member of $\mathcal{M}$. This contradiction shows that $G_{2}$ is isochromatic.

Now we prove that the isochromatic component produced by an arbitrary cycle-separating 4-edge-cut has only eight vertices. Suppose to the contrary that there exists a cycle separating edge-cut $R$ in $G$ such that the isochromatic component, denoted by $J$, has at least ten vertices. Choose $R$ to minimise the number of vertices of $J$. Clearly, the other component $K$ of $G-R$ has at most 34 vertices and, by the first part of the proof, it is uncolourable. It follows that $K$ also has at least ten vertices, so $J$ has at most 34 vertices too. Theorem 5 further implies that $K$ can be extended to a cyclically 4-edge-connected snark $\tilde{K}$ by adding two vertices. If $\zeta(J) \geqslant 3$, the same theorem implies that $J$ can also be extended to a cyclically 4 -edge-connected snark $\tilde{J}$ by adding two vertices. Since both $\tilde{J}$ and $\tilde{K}$ have order at most $36, G$ belongs to $\mathcal{M}-$ a contradiction. Therefore $J$ has a 2-edge-cut. By Theorem 5 (iii), $J$ is a partial junction of two colour-open 4-poles $J_{1}$ and $J_{2}$. Since both $\delta_{G}\left(J_{1}\right)$ and $\delta_{G}\left(J_{2}\right)$ are cycle-separating 4-edge-cuts, both $J_{1}$ and $J_{2}$ must be isochromatic. If any of them had more than eight vertices, then the corresponding edge-cut would contradict the choice of $R$. Therefore $J$ is a partial junction of two copies of the isochromatic 4-pole $\mathbf{I}$ on eight vertices, so $J$ has 16 vertices and $K$ has 28 vertices. It follows that $G$ can be expressed in the form $(P \odot P) \otimes H$ where $P$ is the Petersen graph, $H$ is a snark on 30 vertices, and $L_{1} \odot L_{2}$ denotes a 4-join of cubic graphs $L_{1}$ and $L_{2}$ which employs 4-poles resulting from the removal of two adjacent vertices from both $L_{1}$ and $L_{2}$, while $Q_{1} \otimes Q_{2}$ denotes a 4 -join of cubic graphs $Q_{1}$ and $Q_{2}$ which employs a 4-pole obtained from $Q_{1}$ by removing two nonadjacent edges and a 4-pole obtained from $Q_{2}$ by removing two adjacent vertices. Using a computer we have constructed all graphs $G$ arising in this way and verified that each of them either belongs to $\mathcal{M}$ or has oddness at most 2. This contradiction establishes Claim 1.

Claim 2. Every 4-edge-cut in $G$ separates a subgraph with at most eight vertices from the rest of $G$.

Proof of Claim 2. Let $Q$ be a 4-edge-cut in $G$. If one of the components of $G-Q$ is acyclic, then, by Lemma 1, this component has two vertices. If $Q$ is cycle-separating, then the conclusion follows from Claim 1. Claim 2 is proved.

Fix a cycle-separating 4-edge-cut $S$ in $G$; we will refer to $S$ as the principal 4-edge-cut of $G$. As before, let $G_{1}$ and $G_{2}$ be the components of $G-S$ where $G_{1}$ is uncolourable. By Claim 1, the other component $G_{2}$ is isochromatic on eight vertices. Let $A$ be the set of end-vertices of $S$ in $G_{1}$. Since $S$ is independent, we have $|A|=4$. 
The remainder of the proof is devoted to proving the following fact:

The component $G_{1}$ can be extended to a cyclically 4-edge-connected snark by adding two edges between the vertices of $A$.

As a consequence, $G$ will be a 4 -join of two graphs from $\mathcal{S}_{36}$, and therefore a member of $\mathcal{M}$. This will provide a final contradiction.

Claim 3. $G_{1}$ is cyclically 3-edge-connected and has a cycle-separating 3-edge-cut.

Proof of Claim 3. If $\zeta\left(G_{1}\right) \geqslant 4$, then adding to $G_{1}$ two edges joining the vertices of $A$ in an arbitrary manner would produce a cyclically 4-edge-connected snark of order 36 . Consequently, $G$ would belong to $\mathcal{M}$. Therefore $\zeta\left(G_{1}\right) \leqslant 3$. To prove that $\zeta\left(G_{1}\right) \geqslant 3$ suppose to the contrary that $G_{1}$ has a cycle-separating 2-edge-cut $U$, and let $G_{11}$ and $G_{12}$ be the two components of $G_{1}-U$. Since $G$ is cyclically 4-edge-connected, two edges of $S$ join $G_{2}$ to $G_{11}$ and other two edges of $S$ join $G_{2}$ to $G_{12}$. Thus $\delta_{G}\left(G_{11}\right)$ is a cycle-separating 4-edge-cut which separates $G_{11}$ from $G_{12} \cup G_{2}$. Since $G_{12} \cup G_{2}$ contains more then eight vertices, Claim 1 implies that $G_{11}$ is an isochromatic dipole on eight vertices. Likewise, $G_{12}$ is an isochromatic dipole on eight vertices. Put together, $G$ has altogether 24 vertices, which is again a contradiction. Therefore $\zeta\left(G_{1}\right)=3$. Finally, $G_{1}$ is homeomorphic to a certain cubic graph $\bar{G}_{1}$ with $\zeta\left(\bar{G}_{1}\right)=\zeta\left(G_{1}\right)$ on 32 vertices, so $G_{1}$ is not a subdivision of the complete graph $K_{4}$ and therefore contains a cycle-separating 3-edge-cut. This establishes Claim 3.

Before we can proceed we need two definitions. First, a cycle-separating 3-edge-cut $Q$ in $G_{1}$ will be called balanced if each component of $G_{1}-Q$ is incident with exactly two edges of the principal 4-edge-cut $S$. Otherwise, $Q$ will be called unbalanced. Second, let $R$ and $T$ be two cycle-separating 3-edge-cuts in $G_{1}$. Let $X_{1}$ and $X_{2}$ be the components of $G_{1}-R$ and let $Y_{1}$ and $Y_{2}$ be the components of $G_{1}-T$. The edge-cuts $R$ and $T$ in $G_{1}$ will be called comparable if $X_{i} \subseteq Y_{j}$ or $Y_{j} \subseteq X_{i}$ for some $i, j \in\{1,2\}$.

Claim 4. $G_{1}$ contains two incomparable balanced 3-edge-cuts.

Proof of Claim 4. Let us first observe that if $Q$ is an arbitrary unbalanced 3-edge-cut in $G_{1}$, then adding any two edges between the vertices of $A$ in an arbitrary manner will produce a cubic graph $\tilde{G}_{1}$ where $Q$ has ceased to be an edge-cut. It follows that if every cycle-separating 3-edge-cut in $G_{1}$ is unbalanced, then $\tilde{G}_{1}$ is a cyclically 4-edge-connected snark and $G$ is a 4 -join of $\tilde{G}_{1}$ with the Petersen graph. Since $G_{1}$ has 36 vertices, $G \in \mathcal{M}$ and we have arrived at a contradiction. Therefore $G_{1}$ must contain a balanced 3-edge-cut.

If every pair of balanced 3-edge-cuts in $G_{1}$ is comparable, we can arrange the balanced 3 -edge-cuts in an increasing linear order, say $Q_{1}, \ldots, Q_{m}$. Clearly, there is a component $H_{1}$ of $G_{1}-Q_{1}$ and a component $H_{m}$ of $G_{1}-Q_{m}$ such that both of them are disjoint from all of $Q_{1}, \ldots, Q_{m}$. It is easy to see that $H_{1}$ contains two vertices of $A$ while $H_{m}$ contains the other two, see Figure 14. Thus we can connect the two vertices of $H_{1} \cap A$ to those of $H_{m} \cap A$ by two edges, producing a cyclically 4-edge-connected snark $\tilde{G}_{1}$. Again, $G$ is a 4 -join of $\tilde{G}_{1}$ with the Petersen graph, so $G$ belongs to $\mathcal{M}$ contrary to the assumption. Therefore $G_{1}$ contains two incomparable balanced 3-edge-cuts. This proves Claim 4. 


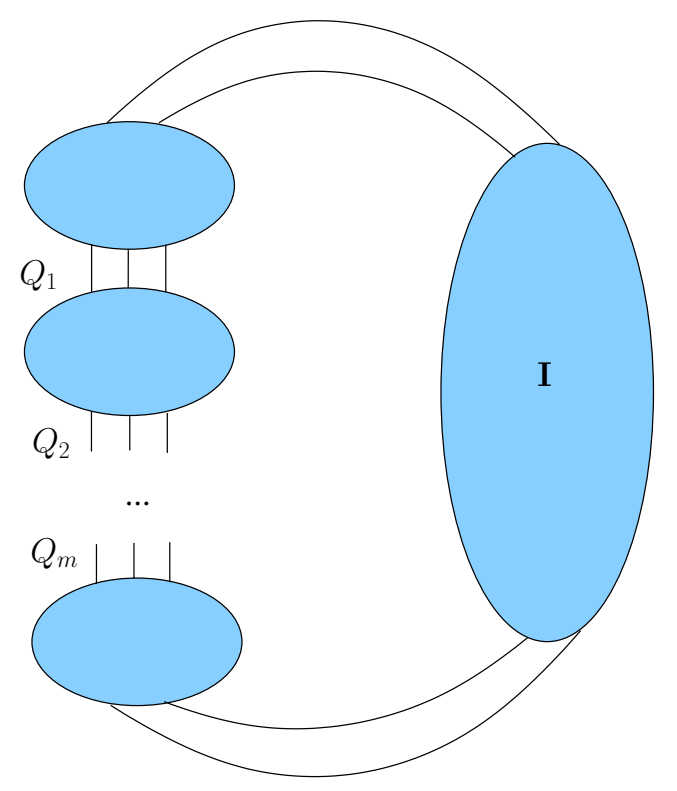

Figure 14: Comparable balanced 3-edge-cuts

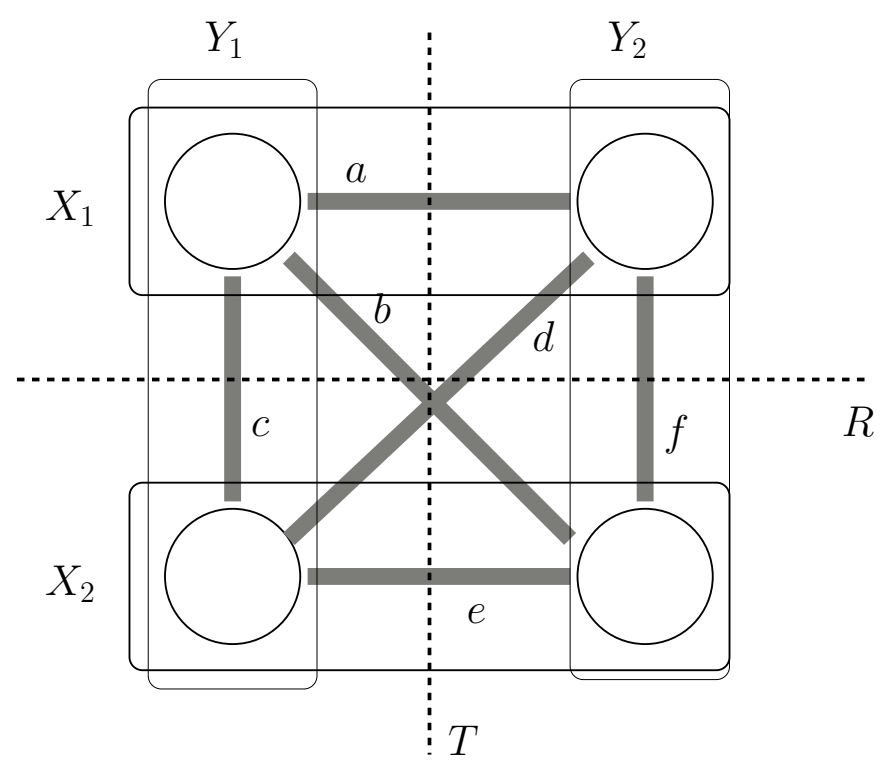

Figure 15: Crossing edge-cuts $R$ and $T$ 
In the rest the proof we explore the structure of $G_{1}$ arising from a pair of incomparable balanced 3-edge-cuts. Let $R$ and $T$ be any two incomparable balanced 3-edge-cuts in $G_{1}$. Clearly, $G_{1}-R$ has two components, say $X_{1}$ and $X_{2}$, and $G_{1}-T$ has two components, say $Y_{1}$ and $Y_{2}$. The definition of comparable edge-cuts readily implies that each of the subgraphs $X_{i} \cap Y_{j}$ is non-empty. Let $a$ be the number of edges between $X_{1} \cap Y_{1}$ and $X_{1} \cap Y_{2}, b$ the number of edges between $X_{1} \cap Y_{1}$ and $X_{2} \cap Y_{2}, c$ the number of edges between $X_{1} \cap Y_{1}$ and $X_{2} \cap Y_{1}$, $d$ the number of edges between $X_{1} \cap Y_{2}$ and $X_{2} \cap Y_{1}$, $e$ the number of edges between $X_{2} \cap Y_{1}$ to $X_{2} \cap Y_{2}$, and finally $f$ the number of edges between $X_{1} \cap Y_{2}$ and $X_{2} \cap Y_{2}$; see Figure 15 .

Since $X_{1}, X_{2}, Y_{1}$ and $Y_{2}$ are all connected, we have

$$
a \geqslant 1, \quad e \geqslant 1, \quad c \geqslant 1, \quad f \geqslant 1 \text {. }
$$

Next,

$$
|R|=\left|\delta_{G_{1}}\left(X_{1}\right)\right|=c+f+b+d=3
$$

and

$$
|T|=\left|\delta_{G_{1}}\left(Y_{1}\right)\right|=a+e+b+d=3 .
$$

By combining (1) and (2) we can further conclude that

$$
b+d \leqslant 1
$$

We now consider two cases according to whether there exists a set $X_{i} \cap Y_{j}$ such that $X_{i} \cap X_{j} \cap A=\emptyset$ or not.

Case 1: There exists a subgraph $X_{i} \cap Y_{j}$, with $i, j \in\{1,2\}$, such that $X_{i} \cap X_{j} \cap A=\emptyset$. In view of symmetry we can clearly assume that $X_{1} \cap X_{2} \cap A=\emptyset$. In this situation $X_{1} \cap Y_{2}$ is incident with two edges of $S$, because $R$ is balanced, and $X_{2} \cap Y_{1}$ is incident with the other two edges of $S$, because $T$ is balanced. It follows that $\left|X_{2} \cap Y_{2} \cap A\right|=0$ as well.

With (1) in mind, we first prove that $a=e=c=f=1$. Suppose to the contrary that one of these values is strictly greater than 1 . In view of symmetry we can assume that $a>1$. Now (1) and (3) forces $a=2, e=1, b=0$, and $d=0$, which together with (2) implies that either $c=2$ and $f=1$, or $c=1$ and $f=2$. In the former case, $\left|\delta_{G}\left(X_{2} \cap Y_{2}\right)\right|=b+e+f=2$ contrary to the fact that $G$ is 3-edge-connected, while in the latter case $X_{2} \cap Y_{1}$ is separated from the rest of $G_{1}$ by $c+d+e=2$ edges, contradicting Claim 3. Therefore $a=e=c=f=1$.

Now, (2) and (4) imply that $b+d=1$. However, $b \geqslant 1$ for otherwise $\left|\delta_{G}\left(X_{1} \cap Y_{1}\right)\right|=$ $a+b+c=2$, contrary to the fact that $G$ is 3 -edge-connected. So $b=1$ and $d=0$. It follows that $\left|\delta_{G}\left(X_{1} \cap Y_{1}\right)\right|=a+b+c=3$ and $\left|\delta_{G}\left(X_{2} \cap Y_{2}\right)\right|=b+e+f=3$. Since $G$ is cyclically 4-edge-connected, both $X_{1} \cap Y_{1}$ and $X_{2} \cap Y_{2}$ are acyclic, and therefore, by Lemma 1, both consist of a single vertex. Further, $\left|\delta_{G}\left(X_{1} \cap Y_{2}\right)\right|=2+a+d+f=4$ and $\left|\delta_{G}\left(X_{2} \cap Y_{1}\right)\right|=2+c+d+e=4$, so $\left|X_{1} \cap Y_{2}\right| \geqslant 2$ and $\left|X_{2} \cap Y_{1}\right| \geqslant 2$. By Claim 2, one of 
the components determined by $\delta_{G}\left(X_{1} \cap Y_{2}\right)$ has at most eight vertices. Since the number of vertices of $G$ lying outside $X_{1} \cap Y_{2}$ is

$$
\left|G_{2}\right|+\left|X_{2} \cap Y_{1}\right|+\left|X_{1} \cap Y_{1}\right|+\left|X_{2} \cap Y_{2}\right| \geqslant 8+2+1+1=12,
$$

we conclude that $\left|X_{1} \cap Y_{2}\right| \leqslant 8$. Similarly, $\left|X_{2} \cap Y_{1}\right| \leqslant 8$. Summing up,

$$
\left|G_{1}\right| \leqslant\left|X_{1} \cap Y_{1}\right|+\left|X_{2} \cap Y_{2}\right|+\left|X_{1} \cap Y_{2}\right|+\left|X_{2} \cap Y_{1}\right| \leqslant 1+1+8+8<36,
$$

which contradicts Claim 1. This establishes Case 1.

Case 2: $X_{i} \cap X_{j} \cap A \neq \emptyset$ for each $i, j \in\{1,2\}$. Clearly, this is only possible when each of the subgraphs $X_{i} \cap Y_{i}$ is incident with exactly one edge of the principal 4-edge-cut $S$. We may assume that $A=\left\{a_{1}, a_{2}, a_{3}, a_{4}\right\}$ where $a_{1}$ lies in $X_{1} \cap Y_{1}, a_{2}$ lies in $X_{1} \cap Y_{2}, a_{3}$ lies in $X_{2} \cap Y_{1}$, and $a_{4}$ lies in $X_{2} \cap Y_{2}$.

For each $X_{i} \cap Y_{i}$ we count the number of edges of $R \cup T$ this subgraph is incident with. According to (1) we obtain

$$
a+b+c \geqslant 2, \quad a+d+f \geqslant 2, \quad c+d+e \geqslant 2, \quad b+e+f \geqslant 2 .
$$

If all the inequalities in (5) are strict, then summing the first two of them yields

$$
6 \leqslant 2 a+(b+c+d+f)=2 a+|R|=2 a+3,
$$

whence $a \geqslant 2$. Similarly, summing the latter two inequalities implies that $e \geqslant 2$. But then

$$
|T|=a+b+d+e \geqslant 4
$$

which is a contradiction.

Therefore at least one of the inequalities in (5) holds with equality. Due to symmetry we may assume that $a+b+c=2$. From (1) we now infer that $a=c=1$ and $b=0$. If we plug these values into (2) and (3), we get $d+f=2=d+e$. Since $d \leqslant 1$ on account of (4), we conclude that either $d=e=f=1$, or $d=0$ and $e=f=2$.

First assume that $d=e=f=1$. In this case $\left|\delta_{G}\left(X_{1} \cap Y_{1}\right)\right|=3=\left|\delta_{G}\left(X_{2} \cap Y_{2}\right)\right|$, so $\left|X_{1} \cap Y_{1}\right|=1=\left|X_{2} \cap Y_{2}\right|$ by Lemma 1. Furthermore, $\left|\delta_{G}\left(X_{1} \cap Y_{2}\right)\right|=\left|\delta_{G}\left(X_{2} \cap Y_{1}\right)\right|=4$. By Claim 2, one of the components determined by $\delta_{G}\left(X_{1} \cap Y_{2}\right)$ has at most eight vertices. As in Case 1, the number of vertices of $G$ outside $X_{1} \cap Y_{2}$ is at least 12 , so $\left|X_{1} \cap Y_{2}\right| \leqslant 8$. Similarly $\left|X_{2} \cap Y_{1}\right| \leqslant 8$, and therefore $\left|G_{1}\right| \leqslant 1+1+8+8<36$, which contradicts Claim 1 .

Next assume that $d=0$ and $e=f=2$. Recall that $a=c=1$ and $b=0$. It follows that $\left|\delta_{G}\left(X_{1} \cap Y_{1}\right)\right|=3$ which means that $X_{1} \cap X_{1}$ consists of a single vertex $a_{1}$. Further, $\left|\delta_{G}\left(X_{1} \cap Y_{2}\right)\right|=4$ and $\left|\delta_{G}\left(X_{2} \cap Y_{1}\right)\right|=4$, so $\left|X_{1} \cap Y_{2}\right| \leqslant 8$ and $\left|X_{2} \cap Y_{1}\right| \leqslant 8$. Also, $\left|\delta_{G}\left(X_{2} \cap Y_{2}\right)\right|=5$. In fact, Claim 1 implies that $X_{1} \cap Y_{2}$ and $X_{2} \cap Y_{1}$ are isochromatic 4poles on eight vertices, because $R$ and $T$ are cycle-separating edge-cuts and hence $X_{1} \cap Y_{2}$ and $X_{2} \cap Y_{1}$ must contain circuits.

We show that $X_{2} \cap Y_{2}$ is 2-edge-connected. If $X_{2} \cap Y_{2}$ was disconnected, it would have a component $B$ with $\left|\delta_{G}(B)\right| \leqslant 2$, contradicting the fact that $G$ is 3 -edge-connected. If 
there was a bridge in $X_{2} \cap Y_{2}$, then the bridge would join a subgraph $B_{1}$ with $\delta_{G}\left(B_{1}\right)=3$ to a subgraph $B_{2}$ with $\delta_{G}\left(B_{2}\right)=4$. By Lemma 1 and Claim $2,\left|B_{1}\right|=1$ while $\left|B_{2}\right| \leqslant 8$, so $\left|G_{1}\right| \leqslant 1+8+8+1+8<36$, contrary to Claim 1 . Therefore $X_{2} \cap Y_{2}$ is 2-edge-connected.

Let us now extend $G_{1}$ to a cubic graph $\tilde{G}_{1}$ by adding the edges $a_{1} a_{4}$ and $a_{2} a_{3}$. Since $G_{1}$ is uncolourable, $\tilde{G}_{1}$ is a snark. We wish to show that $\tilde{G}_{1}$ is cyclically 4-edge-connected. To this end, observe that a cycle-separating 3-edge-cut of $G_{1}$ that separates either $a_{1}$ from $a_{4}$ or $a_{2}$ from $a_{3}$ fails to be an edge-cut in $\tilde{G}_{1}$. Therefore every cycle-separating 3 -edge-cut that might survive from $G_{1}$ to $\tilde{G}_{1}$ separates $\left\{a_{1}, a_{4}\right\}$ from $\left\{a_{2}, a_{3}\right\}$. We prove that such a cut does not exist, displaying four edge-disjoint $\left\{a_{1}, a_{4}\right\}-\left\{a_{2}, a_{3}\right\}$-paths in $G_{1}$.

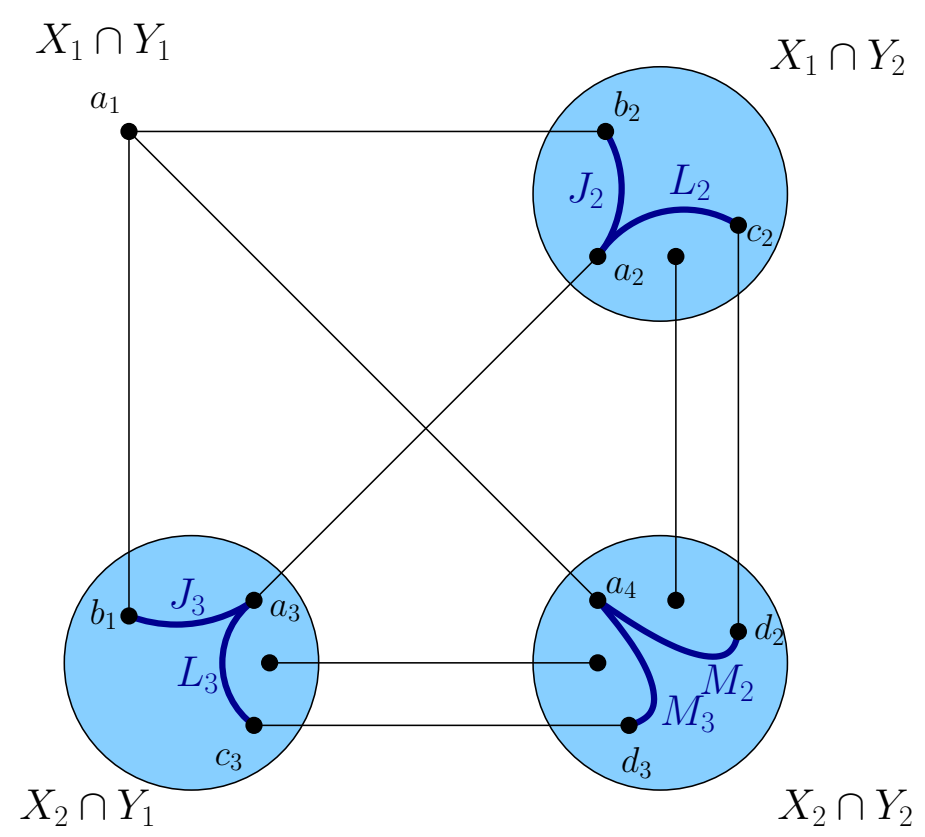

Figure 16: The required paths in Case 2

Let $a_{1} b_{2}$ and $a_{1} b_{3}$ be the edges that join $a_{1}$ to a vertex $b_{2}$ in $X_{1} \cap Y_{2}$ and to a vertex $b_{3}$ in $X_{2} \cap Y_{1}$, respectively. Let $c_{2} d_{2}$ be an edge joining a vertex $c_{2}$ in $X_{1} \cap Y_{2}$ to a vertex $d_{2}$ in $X_{2} \cap Y_{2}$, and let $c_{3} d_{3}$ be an edge joining a vertex $c_{3}$ in $X_{2} \cap Y_{1}$ to a vertex $d_{3}$ in $X_{2} \cap Y_{2}$ (see Figure 16). Since $X_{1} \cap Y_{2}, X_{2} \cap Y_{1}$, and $X_{2} \cap Y_{2}$ are all 2-edge-connected, there exist

- edge-disjoint paths $J_{2}$ and $L_{2}$ in $X_{1} \cap Y_{2}$ from $a_{2}$ to $b_{2}$ and $c_{2}$, respectively;

- edge-disjoint paths $J_{3}$ and $L_{3}$ in $X_{2} \cap Y_{1}$ from $a_{3}$ to $b_{3}$ and $c_{3}$, respectively; and

- edge-disjoint paths $M_{2}$ and $M_{3}$ from $a_{4}$ to $d_{2}$ and $d_{3}$, respectively.

It follows that $a_{1} b_{2} J_{2}^{-1}, a_{1} b_{3} J_{3}^{-1}, L_{2} c_{2} d_{2} M_{2}^{-1}$, and $L_{3} c_{3} d_{3} M_{3}^{-1}$ are four edge-disjoint paths joining $\left\{a_{1}, a_{4}\right\}$ to $\left\{a_{2}, a_{3}\right\}$ in $G_{1}$. As a consequence, $\tilde{G}_{1}$ is a cyclically 4-edge-connected snark of order 36 , and therefore $G \in \mathcal{M}$. This final contradiction establishes the theorem. 


\section{$5 \quad$ Further computational results}

In addition to determining the complete set $\mathcal{M}$ of snarks of oddness at least 4 with cyclic connectivity 4 and minimum number of vertices, we have also generated a considerable number of snarks of oddness at least 4 with orders ranging from 46 to 52 . In order to produce as many nonisomorphic snarks as possible we have applied the 4 -join operation in all possible ways to any pair of cyclically 4-edge-connected snarks with at most 36 vertices as long as the resulting graph had at most 52 vertices and computed its oddness. To extend the set we have further applied I-extensions and I-reductions in all possible ways to the snarks of oddness at least 4 constructed by 4 -joins until no new snarks of oddness at least 4 were found. An I-extension of a cubic graph subdivides two edges $e$ and $f$ of $G$ with a new vertex $v_{e}$ and $v_{f}$, respectively, and adds a new edge between $v_{e}$ and $v_{f}$; an I-reduction is the inverse of an I-extension. The combination of 4 -joins, Iextensions, and I-reductions produced a set 887152 nonisomorphic snarks of orders from 46 to 52 , all of oddness 4 . None of the produced snarks had oddness greater than 4 or cyclic connectivity greater than 4 .

The counts of the number of snarks of oddness at least 4 which our method yielded can be found in Table 3. While the set of 31 snarks of order 44 is complete by Theorem 4 , it is very unlikely that this is the case for any of the sets of snarks of oddness 4 of orders between 46 and 52 .

\begin{tabular}{|c|c|c|c|}
\hline Order & Girth 4 & Girth 5 & Total \\
\hline 44 & 0 & 31 & 31 \\
\hline 46 & $\geqslant 0$ & $\geqslant 484$ & $\geqslant 484$ \\
\hline 48 & $\geqslant 1112$ & $\geqslant 4793$ & $\geqslant 5905$ \\
\hline 50 & $\geqslant 27720$ & $\geqslant 39270$ & $\geqslant 66990$ \\
\hline 52 & $\geqslant 457285$ & $\geqslant 356488$ & $\geqslant 813773$ \\
\hline
\end{tabular}

Table 3: Counts of the smallest snarks of oddness 4 and cyclic connectivity 4 .

In [16, Theorem 12] it was shown that if we allow trivial snarks, the smallest one with oddness greater than 2 has 28 vertices and oddness 4 . There are exactly three such snarks, one with cyclic connectivity 3 and two with cyclic connectivity 2 , all three having girth 5 (see [9] and [16, Corrigendum]). Since small snarks with oddness 4 - irrespectively of their connectivity - can be useful for a better understanding of oddness, we include Table 4 which lists the counts of all trivial snarks of oddness 4 with girth at least 4 of orders from 28 to 34 . The graphs from Table 4 were generated by the first author in [9]. Restricting to girth at least 4 is reasonable because every snark with oddness 4 that contains a triangle or a digon must arise from a smaller snark with the same oddness in a straightforward manner [16, Lemma 1].

A glance at Table 3 reveals that no snarks of oddness 4 and girth 4 on 46 vertices have been found. A similar phenomenon occurs for the trivial snarks in Table 4: there are no 


\begin{tabular}{r|rrr|rrr}
\hline \multirow{2}{*}{ Order } & \multicolumn{2}{|c}{ Girth 4 } & \multicolumn{4}{c}{ Girth 5 } \\
& Connectivity 2 & Connectivity 3 & Total & Connectivity 2 & Connectivity 3 & Total \\
\hline 28 & 0 & 0 & 0 & 2 & 1 & 3 \\
30 & 0 & 0 & 0 & 9 & 4 & 13 \\
32 & 24 & 11 & 35 & 33 & 21 & 54 \\
34 & 315 & 175 & 490 & 139 & 138 & 277 \\
\hline
\end{tabular}

Table 4: Counts of the smallest (trivial) snarks of oddness 4 .

snarks of oddness 4 and girth 4 of order 30. In contrast to orders 44 and 28, we do not have any theoretical argument that would exclude the existence of such snarks.

The graphs from Table 3 can be downloaded from the House of Graphs [3] at http: //hog.grinvin.org/Snarks and the snarks of oddness 4 on 44 and 46 vertices from Table 3 can be inspected at the database of interesting graphs from the House of Graphs by searching for the keywords "nontrivial snarks * oddness 4".

In the remainder of this section, we discuss several invariants of the graphs from Tables 3 and 4, again divided into two groups - uncolourability measures and general invariants. All invariant values have been computed by two independent programs or by programs which were already extensively tested in earlier research. For example, the two independent programs that we have used to compute the oddness of the graphs can be downloaded from [10]. In the Appendix we describe how some of the graphs from Table 3 which appear particularity interesting can be obtained.

\subsection{Resistance and other measures of uncolourability}

We begin our discussion with invariants which, in a certain sense, measure uncolourability of cubic graphs. For all snarks from Tables 3 and 4 we have determined their resistance, perfect matching index, and the invariants $\gamma_{2}$ and $\mu_{3}$. All of them have been defined earlier in this paper and their values were discussed for the 31 snarks in $\mathcal{M}$ in Section 3. We also know that all snarks from Tables 3 and 4 have weak oddness 4 because this is true in general for all snarks of oddness 4 . The remaining invariants have been computed with the help of a computer.

We start with the resistance of the snarks that we have constructed.

Observation 2. All snarks from Table 3 and 4 have resistance 3, except for six snarks on 52 vertices, which have resistance 4.

The first example of a cyclically 4-edge-connected snark with resistance 4 on 52 vertices was constructed by Lukot'ka et al. [16, Section 7]. The same snark is depicted and discussed by Jin and Steffen in [15, Figure 3]. Two more examples of order 52 can be easily obtained if we replace one or both copies of $\mathbf{H}_{1}$ contained in this snark with $\mathbf{H}_{2}$; all three of them are included among the mentioned six snarks. Since we have no guarantee that these are the smallest nontrivial snarks with resistance 4, we offer the following problem. 
Problem 1. What is the smallest order of a snark with resistance 4? What is the smallest order of a nontrivial snark with resistance 4 ?

A snark with oddness 4 and connectivity 2 on 40 vertices was constructed in [16, Section 6]. We have verified that its resistance is 4 , so the upper bound for the first question in Problem 1 is 40. On the other hand, Observation 2 implies that the lower bound is 36 .

All nontrivial snarks with resistance 3 known to us have weak oddness 4 (and hence oddness 4 as well). It is therefore tempting to ask the following questions.

Problem 2. Does there exist a nontrivial snark with resistance 3 and weak oddness greater than 4? Can the difference be arbitrarily large?

Allie [1] constructed nontrivial snarks demonstrating that the difference $\omega-\omega^{\prime}$ can be arbitrarily large. Unfortunately, all of them have resistance 3 and weak oddness 4 while their oddness is arbitrarily large.

The remaining three uncolourability measures that we are going to examine are related to the structure of perfect matchings in snarks: the perfect matching index $\pi$ and the invariants $\mu_{3}$ and $\gamma_{2}$ (see Section 3). The results for perfect matching index are collected in the following observation and Table 5.

Observation 3. All snarks of oddness 4 from Table 3 have perfect matching index $\pi=4$, except for one graph of order 52 which has $\pi=5$.

\begin{tabular}{crrr}
\hline Order & $\pi=4$ & $\pi=5$ & Total \\
\hline 28 & & 3 & 3 \\
30 & & 13 & 13 \\
32 & 6 & 83 & 89 \\
34 & 40 & 727 & 767 \\
\hline
\end{tabular}

Table 5: Counts of the value of perfect matching index for the snarks from Table 4.

While the previous observation seems to suggest that among snarks with oddness at least 4 those with perfect matching index 5 (or more) are rare, Table 5 draws a different picture: only 46 among the 872 trivial snarks of oddness 4 of orders 28-34 listed in Table 4 have perfect matching index 4.

We proceed to the invariant $\mu_{3}$. Tables 6 and 7 show the counts of the value of $\mu_{3}$ of the snarks of oddness 4 from Tables 3 and 4, respectively. Again, there is a striking difference between the trivial and the nontrivial snarks. Most cyclically 4-edge-connected snarks in Table 3 have $\mu_{3}=6$, which is the minimal possible value for snarks with $\omega=4$, according to [15, Corollary 2.4]. By contrast, most trivial snarks from Table 4 have $\mu_{3}>6$.

Finally, we discuss the invariant $\gamma_{2}$. 


\begin{tabular}{crrrrr}
\hline Order & $\mu_{3}=6$ & $\mu_{3}=7$ & $\mu_{3}=8$ & $\mu_{3}=9$ & Total \\
\hline 44 & 31 & & & & 31 \\
46 & 481 & 1 & 2 & & 484 \\
48 & 5878 & 1 & 26 & & 5905 \\
50 & 66724 & 5 & 261 & & 66990 \\
52 & 809349 & 2213 & 2181 & 30 & 813773 \\
\hline
\end{tabular}

Table 6: Counts of the value of $\mu_{3}$ for the snarks from Table 3.

\begin{tabular}{crrrrr}
\hline Order & $\mu_{3}=6$ & $\mu_{3}=7$ & $\mu_{3}=8$ & $\mu_{3}=9$ & Total \\
\hline 28 & 2 & 1 & & & 3 \\
30 & 2 & 4 & 4 & 3 & 13 \\
32 & 18 & 21 & 27 & 23 & 89 \\
34 & 118 & 145 & 239 & 265 & 767 \\
\hline
\end{tabular}

Table 7: Counts of the value of $\mu_{3}$ for the snarks from Table 4 .

Observation 4. All snarks from Table 3 have $\gamma_{2}=2$, except for 30 graphs of order 52 which have $\gamma_{2}=3$.

The 30 snarks with $\gamma_{2}=3$ from Observation 4 are the same graphs as the 30 snarks with $\mu_{3}=9$ from Table 6 .

\begin{tabular}{crrr}
\hline Order & $\gamma_{2}=2$ & $\gamma_{2}=3$ & Total \\
\hline 28 & 3 & & 3 \\
30 & 10 & 3 & 13 \\
32 & 66 & 23 & 89 \\
34 & 522 & 245 & 767 \\
\hline
\end{tabular}

Table 8: Counts of the value of $\gamma_{2}$ for the snarks from Table 4.

Table 8 shows the counts of the value of $\gamma_{2}$ of the snarks of oddness 4 from Table 4 . Again, the picture for trivial snarks is quite different. Most cyclically 4-edge-connected snarks from Table 3 have $\gamma_{2}=2$, which is the minimum possible value for snarks with $\omega=4$, by [27, Proposition 2.1]. By contrast, for trivial snarks the distribution of values $\gamma_{2}=2$ and $\gamma_{2}=3$ is more even.

\subsection{Circumference}

Every hamiltonian cubic graph is 3-edge-colourable, which means that the circumference of every snark can be at most $n-1$, where $n$ denotes the number of vertices. The 
circumference deficit, denoted here by $\xi$, is the difference between order and circumference. It is not difficult to observe that the circumference deficit of a cubic graph $G$ is at least its resistance $\rho(G)$, if the circumference deficit is even, and at least $\rho(G)-1$, otherwise. In this sense, circumference deficit can also be considered as one of the measures of uncolourability of cubic graphs.

The well known dominating cycle conjecture [6] implies that every cyclically 4-edgeconnected snark has circumference at least $4 n / 3$, where $n$ is the order of the graph. On the other hand, in [18] Máčajová and Mazák constructed a family of cyclically 4-edgeconnected snarks on $8 m$ vertices with circumference $7 m+2$. They also made a conjecture that every cyclically 4 -edge-connected cubic has circumference at least $7 n / 8$.

Brinkmann et al. determined in [4] that nearly all nontrivial snarks up to 36 vertices have circumference $n-1$ and the remainder (about $0.002 \%$ ) has circumference $n-2$. We have determined the circumference of our snarks of oddness 4 , and the result is as follows.

Observation 5. All snarks of oddness 4 from Table 3 have circumference deficit 3, except for nine graphs on 52 vertices which have circumference deficit 4.

The nine graphs with circumference deficit $\xi=4$ from Observation 5 include the six graphs of resistance 4 from Observation 2.

As can be seen from Table 9, the behaviour of circumference on trivial snarks is again quite different from that of the nontrivial ones.

\begin{tabular}{crrrr}
\hline Order & $\xi=3$ & $\xi=4$ & $\xi=12$ & Total \\
\hline 28 & 3 & & & 3 \\
30 & 13 & & & 13 \\
32 & 88 & & 1 & 89 \\
34 & 760 & 5 & 2 & 767 \\
\hline
\end{tabular}

Table 9: Counts of the circumference deficit of the snarks from Table 4.

\subsection{Automorphism group}

Tables 10 and 11 show statistics of the number of automorphisms of the snarks from Tables 3 and 4, respectively. With the exception of two snarks on 50 vertices, the automorphism group of each of these graphs has order a power of 2 , three times a power of 2 , or nine times a power of 2 , powers of 2 significantly prevailing.

\subsection{Genus}

Tables 12 and 13 show the genus of the snarks of oddness 4 from Tables 3 and 4 and reveal that among the constructed snarks of oddness 4 there are no snarks of genus smaller than their resistance and no nontrivial snarks of genus smaller than their oddness. On the other hand, it is known that there exist infinitely many snarks of genus 1 (Vodopivec [28]) 


\begin{tabular}{|c|c|c|c|c|c|c|c|c|c|c|c|}
\hline Order & 1 & 2 & 4 & 8 & 10 & 16 & 32 & 64 & 128 & 256 & Total \\
\hline 44 & 8 & 8 & 9 & 3 & & 2 & & 1 & & & 31 \\
\hline 46 & 190 & 105 & 136 & 40 & & 9 & 3 & 1 & & & 484 \\
\hline 48 & 2480 & 1361 & 1602 & 353 & & 93 & 10 & 5 & 1 & & 5905 \\
\hline 50 & 29809 & 15088 & 18032 & 3367 & 2 & 585 & 87 & 20 & & & 66990 \\
\hline 52 & 382025 & 171584 & 221499 & 33586 & & 4350 & 629 & 84 & 12 & 4 & 813773 \\
\hline
\end{tabular}

Table 10: Counts of the order of the automorphism group of the snarks from Table 3.

\begin{tabular}{crrrrrrrrrrrrrrr}
\hline Order & 4 & 8 & 16 & 24 & 32 & 48 & 64 & 96 & 128 & 192 & 256 & 288 & 384 & 768 & Total \\
\hline 28 & & & & & 1 & 1 & & 1 & & & & & & 3 \\
30 & 1 & 2 & & 3 & & 5 & & & & & 1 & 1 & & 13 \\
32 & & 12 & 26 & & 29 & 1 & 14 & 1 & 5 & & & & & 1 & 89 \\
34 & 6 & 198 & 267 & 1 & 183 & 3 & 81 & 2 & 21 & 1 & 3 & & 1 & & 767 \\
\hline
\end{tabular}

Table 11: Counts of the order of the automorphism group of the snarks from Table 4 .

and, in fact, infinitely many snarks of any given genus $g \geqslant 1$ (Mohar and Vodopivec [19, Theorem 2.1]). It is therefore tempting to ask the following two questions.

\begin{tabular}{rrrrr}
\hline Order & Genus 4 & Genus 5 & Genus 6 & Total \\
\hline 44 & 13 & 18 & & 31 \\
46 & 42 & 442 & & 484 \\
48 & 150 & 5713 & 42 & 5905 \\
50 & 531 & 61642 & 4817 & 66990 \\
52 & 2767 & 595528 & 215478 & 813773 \\
\hline
\end{tabular}

Table 12: Counts of the genus of the snarks from Table 3.

\begin{tabular}{crrr}
\hline Order & Genus 3 & Genus 4 & Total \\
\hline 28 & 3 & & 3 \\
30 & 11 & 2 & 13 \\
32 & 54 & 35 & 89 \\
34 & 283 & 484 & 767 \\
\hline
\end{tabular}

Table 13: Counts of the genus of the snarks from Table 4 .

Problem 3. Does there exist a nontrivial snark of oddness $\omega \geqslant 4$ with genus smaller than $\omega$ ? 
Problem 4. Does there exist a snark of resistance $\rho \geqslant 3$ with genus smaller than $\rho$ ?

For $\rho=2$, the questions posed in Problems 3 and 4 have a positive answer, see [19, 28].

\section{Final remarks}

It is important to emphasise that Theorem 4, which is the main result of our previous paper [8], does not yet determine the order of a smallest nontrivial snark with oddness at least 4 . The reason is that it does not exclude the existence of cyclically 5-edge-connected snarks with oddness at least 4 on fewer than 44 vertices. However, the smallest currently known cyclically 5-edge-connected snark with oddness at least 4 has 76 vertices (see [16, Section 8]), which indicates that a cyclically 5-edge-connected snark with oddness at least 4 on fewer than 44 vertices either does not exist or will be very difficult to find. Thus the following problem remains open.

Problem 5. Determine the smallest order of a nontrivial snark with oddness at least 4.

If we take into account the fact that the validity of the cycle double cover conjecture is open for snarks of oddness 6 [11], the following problem appears to be interesting.

Problem 6. Determine the smallest order of a nontrivial snark with oddness at least 6 .

The problem remains interesting even in the version where snarks are required only to be 2-connected rather than nontrivial, since trivial snarks with large oddness can serve as ingredients for constructions of nontrivial ones. The best current upper bound for Problem 6 is 70 in the nontrivial version, see [16, Section 7] and 40 in the trivial version [16, Section 6]. It follows from [9] that the current lower bound for the trivial version is 36 .

\section{Acknowledgements}

We would like to thank Gunnar Brinkmann for providing us with an independent program for computing the genus of a graph. Most of the computations were carried out using the Stevin Supercomputer Infrastructure at Ghent University.

\section{References}

[1] I. Allie, Oddness to resistance ratios in cubic graphs, Discrete Math. 342 (2019), 387-392

[2] A. Bonisoli and D. Cariolaro, Excessive factorizations of regular graphs, in: Graph Theory in Paris, Birkhauser, Basel, 2007, pp. 73-84.

[3] G. Brinkmann, K. Coolsaet, J. Goedgebeur, H. Mélot, House of Graphs: a database of interesting graphs, Discrete Appl. Math. 161 (2013), 311-314. Available at http: //hog.grinvin.org/. 
[4] G. Brinkmann, J. Goedgebeur, J. Hägglund, K. Markström, Generation and properties of snarks, J. Combin. Theory Ser. B 103 (2013), 468-488.

[5] M. A. Fiol, G. Mazzuoccolo, E. Steffen, Measures of edge-uncolorability of cubic graphs, Electron. J. Combin. 25 (2018), \#P4.54.

[6] H. Fleischner, Cycle decompositions, 2-coverings, removable cycles and the fourcolor disease, in: J. A. Bondy, U. S. R. Murty (Eds.), Progress in Graph Theory, Academic Press, New York, 1984, pp. 233-246.

[7] J.-L. Fouquet, J.-M. Vanherpe, On the perfect matching index of bridgeless cubic graphs, arXiv:0904.1296 (2009).

[8] J. Goedgebeur, E. Máčajová, M. Škoviera, Smallest snarks with oddness 4 and cyclic connectivity 4 have order 44, Ars Math. Contemp. 16 (2019), 277-298.

[9] J. Goedgebeur, On the smallest snarks with oddness 4 and connectivity 2, Electron. J. Combin. 25 (2018), \#P2.15.

[10] J. Goedgebeur, Source code of two programs to compute the oddness of a graph: http://caagt. ugent. be/oddness/.

[11] R. Häggkvist and S. McGuinness, Double covers of cubic graphs of oddness 4, J. Combin. Theory Ser. B 93 (2005), 251-277.

[12] J. Hägglund, On snarks that are far from being 3-edge-colorable, Electron. J. Combin. 23 (2016), \#P2.6.

[13] A. Huck, M. Kochol, Five cycle double covers of some cubic graphs, J. Combin. Theory Ser. B 64 (1995), 119-125.

[14] F. Jaeger, Nowhere-zero flow problems, in L. W. Beinecke, R. J. Wilson (ed.), Selected topics in graph theory 3, Academic Press, San Diego, CA (1988), 71-95.

[15] L. Jin, E. Steffen, Petersen cores and the oddness of cubic graphs, J. Graph Theory 84 (2017), 109-120.

[16] R. Lukot'ka, E. Máčajová, J. Mazák, M. Škoviera, Small snarks with large oddness, Electron. J. Combin. 22 (2015), \#P1.51.

[17] R. Lukot'ka, J. Mazák, Weak oddness as an approximation of oddness and resistance in cubic graphs, Discrete Appl. Math. 244 (2018), 223-226.

[18] E. Máčajová, J. Mazák, Cubic graphs with large circumference deficit, J. Graph Theory 82 (2016), 433-440.

[19] B. Mohar, A. Vodopivec, The genus of Petersen powers, J. Graph Theory 67 (2011), $1-8$.

[20] R. Nedela, M. Škoviera, Atoms of cyclic connectivity in cubic graphs, Math. Slovaca 45 (1995), 481-499.

[21] R. Nedela, M. Škoviera, Decompositions and reductions of snarks, J. Graph Theory 22 (1996), 253-279.

[22] N. Robertson, Minimal cyclic-4-connected graphs, Trans. Amer. Math. Soc. 284 (1984), 665-687. 
[23] P. D. Seymour, On multi-colourings of cubic graphs and conjectures of Fulkerson and Tutte, Proc. London Math. Soc. 38, 423-460 (1979)

[24] E. Steffen, Classifications and characterizations of snarks, Discrete Math. 188 (1998), 183-203.

[25] E. Steffen, Measurements of edge-uncolorability, Discrete Math. 280 (2004), 191214.

[26] E. Steffen, 1-Factor and cycle covers of cubic graphs, J. Graph Theory 78 (2015), 195-206.

[27] E. Steffen, Intersecting 1-factors and nowhere-zero 5-flows, Combinatorica 35 (2015), 633-640.

[28] A. Vodopivec, On embeddings of snarks in the torus, Discrete Math. 308 (2008), $1847-1849$.

\section{Appendix 1: Obtaining graphs from the Observations in Section 5}

Exceptional graphs mentioned in Observations 2-5 in Section 5 can be obtained from the database of interesting graphs in the House of Graphs [3] by searching keywords "snark * resistance 4", "snark * perfect matching index 5", "snark * gamma", and "snark * circumference deficit 4", respectively.

\section{Appendix 2: Adjacency lists of the 31 snarks in $\mathcal{M}$}

We present the adjacency lists of the 31 snarks in $\mathcal{M}$. These graphs can be downloaded at http://hog.grinvin.org/Snarks or can be inspected at the database of interesting graphs from the House of Graphs [3] by searching for the keywords "nontrivial snarks * oddness 4". The graphs are listed in the order in which they were generated.

1. $\{0: 1214$ 27; 1: 69 16; 2: 49 17; 3: 57 39; 4: 25 13; 5: 34 16; 6: 17 15; 7: 36 17; 8: 1112 15; 9: 12 35; 10: 11 13 14; 11: 810 34; 12: 08 13; 13: 410 12; 14: 0 10 15; 15: 68 14; 16: 15 17; 17: 27 16; 18: 22 26 29; 19: 3136 38; 20: 25 27 42; 21: 23 25 43; 22: 18 23 42; 23: 2122 40; 24: 2532 33; 25: 2021 24; 26: 1827 43; 27: 020 26; 28: 2932 34; 29: 1828 30; 30: 2931 33; 31: 19 30 32; 32: 2428 31; 33: 2430 34; 34: 1128 33; 35: 9 37 38; 36: 1937 39; 37: 35 36 40; 38: 1935 41; 39: 3 36 41; 40: 2337 41; 41: 3839 40; 42: 2022 43; 43: 212642$\} \quad$ (Class 1b)

2. $\{0: 1214$ 38; 1: 69 16; 2: 49 17; 3: 57 22; 4: 25 13; 5: 34 16; 6: 17 15; 7: 36 17; 8: 1112 15; 9: 12 26; 10: 11 13 14; 11: 810 32; 12: 08 13; 13: 410 12; 14: 0 10 15; 15: 68 14; 16: 15 17; 17: 27 16; 18: 21 25 28; 19: 2124 26; 20: 22 24 25; 21: 18 19 22; 22: 320 21; 23: 2431 42; 24: 1920 23; 25: 1820 26; 26: 9 19 25; 27: 3135 43; 28: 1829 43; 29: 2834 40; 30: 3136 39; 31: 2327 30; 32: 1135 37; 33: 3739 40; 34: 29 35 42; 35: 2732 34; 36: 3037 41; 37: 3233 36; 38: 039 41; 39: 3033 38; 40: 29 33 41; 41: 3638 40; 42: 2334 43; 43: 272842$\} \quad$ (Class 4a) 
3. $\{0: 1214$ 28; 1: 69 16; 2: 49 17; 3: 57 24; 4: 25 13; 5: 34 16; 6: 17 15; 7: 36 17; 8: 1112 15; 9: 12 18; 10: 1113 14; 11: 810 36; 12: 08 13; 13: 410 12; 14: 0 10 15; 15: 6 8 14; 16: 15 17; 17: 27 16; 18: 922 25; 19: 3240 42; 20: 22 24 26; 21: 23 24 25; 22: 18 20 23; 23: 2122 40; 24: 3 20 21; 25: 1821 26; 26: 20 25 27; 27: 2629 42; 28: 0 33 34; 29: 2738 41; 30: 3132 37; 31: 3035 36; 32: 1930 33; 33: 2832 43; 34: 28 35 37; 35: 3134 38; 36: 11 31 39; 37: 3034 39; 38: 29 35 39; 39: 3637 38; 40: 1923 41; 41: 29 40 43; 42: $192743 ; 43: 334142\} \quad$ (Class 4a)

4. $\{0: 1214$ 34; 1: 56 9; 2: 47 9; 3: 57 37; 4: 25 13; 5: 13 4; 6: 17 8; 7: 23 6; 8: 611 15; 9: 12 18; 10: 13 14 16; 11: 816 32; 12: 013 17; 13: 410 12; 14: 010 15; 15: 814 17; 16: 1011 17; 17: 1215 16; 18: 922 26; 19: 2830 42; 20: 22 25 27; 21: 23 25 26; 22: 18 20 23; 23: 2122 38; 24: 2529 31; 25: 2021 24; 26: 1821 27; 27: 2026 28; 28 : 1927 40; 29: 2432 35; 30: 1933 35; 31: 2433 34; 32: 11 29 33; 33: 3031 32; 34: 031 35; 35: 29 30 34; 36: 3841 42; 37: 3 39 41; 38: 23 36 39; 39: 3738 43; 40: 2841 43; 41: 3637 40; 42: 1936 43; 43: 394042$\} \quad$ (Class 1b)

5. $\{0: 48$ 12; 1: 2022 32; 2: 79 24; 3: 57 25; 4: 05 24; 5: 34 21; 6: 715 16; 7: 23 6; 8: 09 25; 9: 28 23; 10: 12 15 17; 11: 1920 23; 12: 010 13; 13: 12 14 16; 14: 1315 39; 15: 610 14; 16: 613 17; 17: 1016 26; 18: 1921 22; 19: 1118 37; 20: 111 21; 21: 518 20; 22: 118 23; 23: 911 22; 24: 24 25; 25: 38 24; 26: 17 29 38; 27: 31 32 34; 28: 3033 34; 29: 2631 33; 30: 2831 37; 31: 2729 30; 32: 127 33; 33: 28 29 32; 34: 27 28 36; 35: 37 40 42; 36: 3441 42; 37: 1930 35; 38: 2640 43; 39: 1441 43; 40: 3538 41; 41: 3639 40; 42: $353643 ; 43: 383942\} \quad$ (Class 3a)

6. $\{0: 48$ 32; 1: 1420 22; 2: 47 9; 3: 57 8; 4: 02 5; 5: 34 21; 6: 1516 26; 7: 23 37; 8: 0 3 9; 9: 28 11; 10: 1215 17; 11: 919 23; 12: 1013 39; 13: 12 14 16; 14: 1 13 15; 15: 610 14; 16: 613 17; 17: 1016 19; 18: 2122 24; 19: 11 17 24; 20: 121 25; 21: 5 18 20; 22: 1 18 23; 23: 1122 25; 24: 1819 25; 25: 2023 24; 26: 629 38; 27: 31 32 34; 28: 30 33 34; 29: 2631 33; 30: 2831 37; 31: 2729 30; 32: 027 33; 33: 2829 32; 34: 27 28 36; 35: 37 40 42; 36: 3441 42; 37: 730 35; 38: 2640 43; 39: 1241 43; 40: 3538 41; 41: 3639 40; 42: $353643 ; 43: 383942\} \quad$ (Class 3a)

7. $\{0: 1214$ 41; 1: 56 9; 2: 47 9; 3: 57 30; 4: 25 13; 5: 13 4; 6: 17 8; 7: 23 6; 8: 611 15; 9: 12 19; 10: 13 14 16; 11: 816 43; 12: 013 17; 13: 4 10 12; 14: 010 15; 15: 814 17; 16: 1011 17; 17: 1215 16; 18: 2226 30; 19: 923 31; 20: 22 25 27; 21: 23 25 26; 22: 18 20 23; 23: 1921 22; 24: 2533 35; 25: 2021 24; 26: 1821 27; 27: 2026 38; 28: 2933 36; 29: 2840 42; 30: 318 32; 31: 19 34 35; 32: 3034 36; 33: 2428 34; 34: 31 32 33; 35: 24 31 36; 36: 28 32 35; 37: 3840 43; 38: 2737 39; 39: 38 41 42; 40: 2937 41; 41: 039 40; 42: 2939 43; 43: 113742$\} \quad$ (Class 3b)

8. $\{0: 1214$ 40; 1: 56 9; 2: 47 9; 3: 57 21; 4: 25 13; 5: 13 4; 6: 17 8; 7: 23 6; 8: 611 15; 9: 12 26; 10: 13 14 16; 11: 816 38; 12: 013 17; 13: 410 12; 14: 010 15; 15: 814 17; 16: 1011 17; 17: 1215 16; 18: 2529 33; 19: 2830 31; 20: 2234 42; 21: 322 43; 22: 20 21 31; 23: 2430 32; 24: 2335 36; 25: 1826 42; 26: 9 25 28; 27: 2829 32; 28 : 1926 27; 29: 1827 30; 30: 19 23 29; 31: 1922 32; 32: 2327 31; 33: 1834 43; 34: 20 33 37; 35: 24 38 41; 36: 2439 40; 37: 3439 41; 38: 1135 39; 39: 36 37 38; 40: 036 41; 41: 3537 40; 42: 2025 43; 43: 213342$\} \quad$ (Class 4b)

9. $\{0: 1214$ 29; 1: 69 16; 2: 49 17; 3: 57 35; 4: 25 13; 5: 34 16; 6: 17 15; 7: 36 17; 8: 1112 15; 9: 12 40; 10: 11 13 14; 11: 810 28; 12: 08 13; 13: 410 12; 14: 0 10 15; 15: 68 
14; 16: 15 17; 17: 27 16; 18: 22 26 29; 19: 3137 38; 20: 22 25 27; 21: 23 25 26; 22: 18 20 23; 23: 2122 39; 24: 25 30 32; 25: 2021 24; 26: 18 21 27; 27: 2026 28; 28: 1127 42; 29: 018 33; 30: 2431 36; 31: 1930 34; 32: 2434 35; 33: 2934 36; 34: 31 32 33; 35: 332 36; 36: 30 33 35; 37: 1939 43; 38: 1941 42; 39: 23 37 41; 40: 9 41 43; 41: 3839 40; 42: 2838 43; 43: 374042$\} \quad$ (Class 3c)

10. $\{0: 1214$ 28; 1: 56 9; 2: 47 9; 3: 57 18; 4: 25 13; 5: 13 4; 6: 17 8; 7: 23 6; 8: 611 15; 9: 12 24; 10: 13 14 16; 11: 816 33; 12: 013 17; 13: 410 12; 14: 010 15; 15: 814 17; 16: 1011 17; 17: 1215 16; 18: 322 26; 19: 3034 38; 20: 22 25 27; 21: 23 25 26; 22: 18 20 23; 23: 2122 34; 24: 925 42; 25: 2021 24; 26: 1821 27; 27: 2026 29; 28 : 031 32; 29: 2736 39; 30: 1931 33; 31: 2830 42; 32: 2837 43; 33: 1130 43; 34: 19 23 40; 35: 3739 40; 36: 2937 41; 37: 3235 36; 38: 1939 41; 39: 2935 38; 40: 3435 41; 41: 3638 40; 42: $243143 ; 43: 323342\} \quad$ (Class 2)

11. $\{0: 1214$ 33; 1: 56 9; 2: 47 9; 3: 57 24; 4: 25 13; 5: 13 4; 6: 17 8; 7: 23 6; 8: 611 15; 9: 12 18; 10: 1314 16; 11: 816 28; 12: 013 17; 13: 410 12; 14: 010 15; 15: 814 17; 16: 1011 17; 17 : 1215 16; 18: 922 26; 19: 3034 38; 20: 22 25 27; 21: 23 25 26; 22: 18 20 23; 23: 21 22 34; 24: 325 42; 25: 2021 24; 26: 1821 27; 27: 20 26 29; 28: 1131 32; 29: 2736 39; 30: 1931 33; 31: 2830 42; 32: 2837 43; 33: 030 43; 34: 19 23 40; 35: 37 39 40; 36: 2937 41; 37: 3235 36; 38: 1939 41; 39: 2935 38; 40: 34 35 41; 41: 3638 40; 42: 2431 43; 43: 323342$\} \quad$ (Class 2)

12. $\{0: 121438 ; 1: 56$ 9; 2: 47 9; 3: 57 25; 4: 25 13; 5: 13 4; 6: 17 8; 7: 23 6; 8: 611 15; 9: 12 26; 10: 13 14 16; 11: 816 41; 12: 013 17; 13: 410 12; 14: 010 15; 15: 814 17; 16: 1011 17; 17: 1215 16; 18: 2630 34; 19: 2931 32; 20: 3638 40; 21: 23 35 42; 22: 23 36 43; 23: 2122 32; 24: 2531 33; 25: 324 35; 26: 9 18 42; 27: 2939 40; 28: 2930 33; 29: 1927 28; 30: 1828 31; 31: 1924 30; 32: 1923 33; 33: 2428 32; 34: 18 35 43; 35: 2125 34; 36: 2022 37; 37: 3639 41; 38: 020 39; 39: 27 37 38; 40: 2027 41; 41: 1137 40; 42: 2126 43; 43: 223442$\} \quad$ (Class 4b)

13. $\{0: 314$ 22; 1: 56 9; 2: 47 9; 3: 05 7; 4: 25 13; 5: 13 4; 6: 17 8; 7: 23 6; 8: 611 15; 9: 12 26; 10: 1314 16; 11: 816 29; 12: 1317 30; 13: 410 12; 14: 010 15; 15: 814 17; 16: 1011 17; 17: 1215 16; 18: 21 25 38; 19: 21 24 26; 20: 22 24 25; 21: 18 19 22; 22: 0 20 21; 23: 2437 40; 24: 1920 23; 25: 1820 26; 26: 9 19 25; 27: 3139 41; 28: 3234 37; 29: 1133 34; 30: 12 32 35; 31: 2733 35; 32: 2830 33; 33: 2931 32; 34: 28 29 35; 35: 30 31 34; 36: 3841 42; 37: 2328 42; 38: 18 36 39; 39: 2738 43; 40: 23 41 43; 41: 2736 40; 42: 3637 43; 43: 394042$\} \quad$ (Class 4a)

14. $\{0: 312$ 22; 1: 56 9; 2: 47 9; 3: 05 7; 4: 25 13; 5: 13 4; 6: 17 8; 7: 23 6; 8: 611 15; 9: 12 26; 10: 1314 16; 11: 816 29; 12: 013 17; 13: 410 12; 14: 1015 30; 15: 814 17; 16: 1011 17; 17: 1215 16; 18: 2125 38; 19: 2124 26; 20: 22 24 25; 21: 18 19 22; 22: 0 20 21; 23: 2437 40; 24: 1920 23; 25: 1820 26; 26: 919 25; 27: 3139 41; 28: 3234 37; 29: 1133 34; 30: 1432 35; 31: 2733 35; 32: 2830 33; 33: 2931 32; 34: 28 29 35; 35: 30 31 34; 36: 3841 42; 37: 2328 42; 38: 18 36 39; 39: 2738 43; 40: 23 41 43; 41: 2736 40; 42: $363743 ; 43: 394042\} \quad$ (Class 4a)

15. $\{0: 1214$ 22; 1: 56 9; 2: 47 9; 3: 57 30; 4: 25 13; 5: 13 4; 6: 17 8; 7: 23 6; 8: 611 15; 9: 12 29; 10: 1314 16; 11: 816 26; 12: 013 17; 13: 410 12; 14: 010 15; 15: 814 17; 16: 1011 17; 17: 1215 16; 18: 2125 38; 19: 2124 26; 20: 22 24 25; 21: 18 19 22; 22: 0 20 21; 23: 2437 40; 24: 1920 23; 25: 1820 26; 26: 11 19 25; 27: 3139 41; 28: 3234 37; 
29: 933 34; 30: 332 35; 31: 2733 35; 32: 2830 33; 33: 2931 32; 34: 28 29 35; 35: 3031 34; 36: 3841 42; 37: 2328 42; 38: 1836 39; 39: 2738 43; 40: 2341 43; 41: 2736 40; 42: $363743 ; 43: 394042\} \quad$ (Class 1a)

16. $\{0: 12$ 14 27; 1: 69 16; 2: 49 17; 3: 57 38; 4: 25 13; 5: 34 16; 6: 17 15; 7: 36 17; 8: 11 12 15; 9: 12 41; 10: 11 13 14; 11: 810 35; 12: 08 13; 13: 410 12; 14: 0 10 15; 15: 68 14; 16: 15 17; 17: 27 16; 18: 2630 34; 19: 2931 32; 20: 2538 40; 21: 23 35 42; 22: 23 36 43; 23: 2122 32; 24: 2531 33; 25: 2024 37; 26: 1827 42; 27: 026 29; 28: 2930 33; 29: 1927 28; 30: 1828 31; 31: 1924 30; 32: 1923 33; 33: 2428 32; 34: 1835 43; 35: 11 21 34; 36: 2239 40; 37: 2539 41; 38: 320 39; 39: 36 37 38; 40: 2036 41; 41: 937 40; 42: 2126 43; 43: 2234 42\} (Class 4b)

17. $\{0: 1214$ 38; 1: 69 16; 2: 49 17; 3: 57 30; 4: 25 13; 5: 34 16; 6: 17 15; 7: 36 17; 8: 11 12 15; 9: 12 33; 10: 11 13 14; 11: 810 35; 12: 08 13; 13: 410 12; 14: 0 10 15; 15: 68 14; 16: 15 17; 17: 27 16; 18: 21 25 28; 19: 2426 42; 20: 22 24 43; 21: 1822 42; 22: 20 21 34; 23: 2431 32; 24: 1920 23; 25: 1826 43; 26: 19 25 40; 27: 2831 33; 28: 1827 29; 29: 2830 32; 30: 329 31; 31: 2327 30; 32: 23 29 33; 33: 927 32; 34: 22 36 39; 35: 1137 39; 36: 3437 38; 37: 35 36 40; 38: 036 41; 39: 34 35 41; 40: 2637 41; 41: 3839 40; 42: $192143 ; 43: 202542\} \quad$ (Class 1a)

18. $\{0: 1214$ 38; 1: 69 16; 2: 49 17; 3: 57 30; 4: 25 13; 5: 34 16; 6: 17 15; 7: 36 17; 8: 1112 15; 9: 12 33; 10: 11 13 14; 11: 810 35; 12: 08 13; 13: 410 12; 14: 0 10 15; 15: 68 14; 16: 15 17; 17: 27 16; 18: 21 25 28; 19: 2124 26; 20: 22 24 25; 21: 18 19 22; 22: 20 21 34; 23: 2431 32; 24: 1920 23; 25: 1820 26; 26: 19 25 37; 27: 2831 33; 28: 1827 29; 29: 2830 32; 30: 329 31; 31: 2327 30; 32: 23 29 33; 33: 927 32; 34: 22 36 39; 35: 1137 42; 36: 3438 43; 37: 2635 40; 38: 036 41; 39: 34 41 42; 40: 3741 43; 41: 3839 40; 42: 3539 43; 43: 364042$\} \quad$ (Class 1a)

19. $\{0: 1214$ 29; 1: 69 16; 2: 49 17; 3: 57 38; 4: 25 13; 5: 34 16; 6: 17 15; $7: 36$ 17; 8: 1112 15; 9: 12 36; 10: 11 13 14; 11: 810 23; 12: 08 13; 13: 410 12; 14: 0 10 15; 15: 68 14; 16: 15 17; 17: 27 16; 18: 22 26 30; 19: 3236 40; 20: 22 25 27; 21: 23 25 26; 22: 18 20 23; 23: 1121 22; 24: 2533 34; 25: 2021 24; 26: 1821 27; 27: 2026 29; 28: 3035 42; 29: 027 41; 30: 1828 31; 31: 3032 43; 32: 1931 33; 33: 2432 42; 34: 24 35 43; 35: 28 34 39; 36: 9 19 37; 37: 3639 41; 38: 339 40; 39: 35 37 38; 40: 1938 41; 41: 2937 40; 42: $283343 ; 43: 313442\} \quad$ (Class 2)

20. $\{0: 1214$ 40; 1: 56 9; 2: 47 9; 3: 57 21; 4: 25 13; 5: 13 4; 6: 17 8; 7: 23 6; 8: 611 15; 9: 12 23; 10: 13 14 16; 11: 816 38; 12: 013 17; 13: 4 10 12; 14: 010 15; 15: 814 17; 16: 1011 17; 17: 1215 16; 18: 2428 32; 19: 2729 30; 20: 2233 42; 21: 322 43; 22: 20 21 30; 23: 929 31; 24: 1825 42; 25: 2427 34; 26: 2728 31; 27: 1925 26; 28: 1826 29; 29: 1923 28; 30: 1922 31; 31: 2326 30; 32: 1833 43; 33: 2032 37; 34: 25 35 36; 35: 34 38 41; 36: 3439 40; 37: 3339 41; 38: 1135 39; 39: 36 37 38; 40: 036 41; 41: 3537 40; 42: 2024 43; 43: 213242$\} \quad$ (Class 4b)

21. $\{0: 1214$ 39; 1: 56 9; 2: 47 9; 3: 57 27; 4: 25 13; 5: 13 4; 6: 17 8; 7: 23 6; 8: 611 15; 9: 12 34; 10: 1314 16; 11: 816 35; 12: 013 17; 13: 410 12; 14: 010 15; 15: 814 17; 16: 1011 17; 17: 1215 16; 18: 2226 29; 19: 3136 38; 20: 2527 42; 21: 23 25 43; 22: 18 23 42; 23: 2122 40; 24: 25 32 33; 25: 2021 24; 26: 1827 43; 27: 320 26; 28 : 29 32 34; 29: 1828 30; 30: 2931 33; 31: 19 30 32; 32: 24 28 31; 33: 24 30 34; 34: 9 28 33; 35: 11 37 38; 36: 1937 39; 37: 35 36 40; 38: 1935 41; 39: 036 41; 40: 2337 41; 41: 3839 40; 42: 2022 43; 43: 212642$\} \quad$ (Class 1b) 
22. $\{0: 48$ 12; 1: 2022 32; 2: 79 24; 3: 57 25; 4: 05 24; 5: 34 21; 6: 715 16; 7: 2 3 6; 8: 09 25; 9: 28 23; 10: 12 15 17; 11: 1920 23; 12: 010 13; 13: 12 14 16; 14: 13 15 29; 15: 610 14; 16: 613 17; 17: 1016 35; 18: 1921 22; 19: 1118 33; 20: 111 21; 21: 518 20; 22: 118 23; 23: 911 22; 24: 24 25; 25: 38 24; 26: 33 36 40; 27: 3537 38; 28: 3041 42; 29: 1430 43; 30: 2829 38; 31: 32 37 39; 32: 131 41; 33: 1926 42; 34: 35 36 39; 35: 17 27 34; 36: 26 34 37; 37: 2731 36; 38: 2730 39; 39: 3134 38; 40: 2641 43; 41: 2832 40; 42: 2833 43; 43: 294042$\} \quad$ (Class 4b)

23. $\{0: 48$ 12; 1: 514 38; 2: 47 9; 3: 57 8; 4: 02 5; 5: 13 4; 6: 716 18; 7: 23 6; 8: 03 9; 9: 28 22; 10: 1116 19; 11: 10 13 21; 12: 015 41; 13: 1123 24; 14: 117 18; 15 : 1217 19; 16: 610 17; 17: 1415 16; 18: 614 19; 19: 1015 18; 20: 2123 30; 21: 11 20 25; 22: 923 25; 23: 1320 22; 24: 1325 34; 25: 2122 24; 26: 2933 36; 27: 3234 42; 28: 3032 43; 29: 2630 42; 30: 2028 29; 31: 3239 40; 32: 2728 31; 33: 2634 43; 34: 2427 33; 35: 3639 41; 36: 2635 37; 37: 36 38 40; 38: 137 39; 39: 31 35 38; 40: 3137 41; 41: 12 35 40; 42: 2729 43; 43: 283342$\} \quad$ (Class 3b)

24. $\{0: 1214$ 37; 1: 69 16; 2: 49 17; 3: 57 34; 4: 25 13; 5: 34 16; 6: 17 15; 7: 36 17; 8: 1112 15; 9: 12 32; 10: 11 13 14; 11: 810 18; 12: 08 13; 13: 410 12; 14: 010 15; 15: 68 14; 16: 15 17; 17: 27 16; 18: 1122 26; 19: 2830 42; 20: 22 25 27; 21: 23 25 26; 22: 18 20 23; 23: 2122 38; 24: 2529 31; 25: 2021 24; 26: 1821 27; 27: 2026 28; 28: 1927 40; 29: 2432 35; 30: 1933 35; 31: 2433 34; 32: 929 33; 33: 3031 32; 34: 3 31 35; 35: 2930 34; 36: 3841 42; 37: 039 41; 38: 2336 39; 39: 37 38 43; 40: 2841 43; 41: 3637 40; 42: 1936 43; 43: 394042$\} \quad$ (Class 1b)

25. $\{0: 812$ 16; 1: 1113 14; 2: 718 22; 3: 5 17 24; 4: 525 38; 5: 34 14; 6 : 7 13 15; 7: 26 21; 8: 09 24; 9: 811 41; 10: 11 12 15; 11: 19 10; 12: 0 10 13; 13: 16 12; 14: 15 15; 15: 610 14; 16: 017 25; 17: 3 16 23; 18: 2 19 34; 19: 1821 23; 20: 2122 30; 21 : 719 20; 22: 220 23; 23: 1719 22; 24: 38 25; 25: 416 24; 26: 29 33 36; 27: 32 34 42; 28: 3032 43; 29: 2630 42; 30: 2028 29; 31: 3239 40; 32: 2728 31; 33: 2634 43; 34: 1827 33; 35: 36 39 41; 36: 2635 37; 37: 36 38 40; 38: 437 39; 39: 31 35 38; 40: 3137 41; 41: 935 40; 42: $272943 ; 43: 283342\} \quad$ (Class 4b)

26. $\{0: 1214$ 35; 1: 56 9; 2: 47 9; 3: 57 29; 4: 25 13; 5: 13 4; 6: 17 8; 7: 23 6; 8: 611 15; 9: 12 28; 10: 13 14 16; 11: 816 40; 12: 013 17; 13: 4 10 12; 14: 010 15; 15: 814 17; 16: 1011 17; 17: 1215 16; 18: 2226 29; 19: 3137 38; 20: 22 25 27; 21: 23 25 26; 22: 18 20 23; 23: 2122 39; 24: 2530 32; 25: 2021 24; 26: 1821 27; 27: 2026 28; 28 : 927 42; 29: 318 33; 30: 2431 36; 31: 19 30 34; 32: 24 34 35; 33: 2934 36; 34: 31 32 33; 35: 032 36; 36: 3033 35; 37: 1939 43; 38: 1941 42; 39: 2337 41; 40: 1141 43; 41: 38 39 40; 42: 2838 43; 43: 374042$\} \quad$ (Class 3c)

27. $\{0: 1214$ 18; 1: 69 16; 2: 49 17; 3: 57 28; 4: 2 5 13; 5: 34 16; 6: 17 15; 7: 36 17; 8: 11 12 15; 9: 12 33; 10: 11 13 14; 11: 810 24; 12: 08 13; 13: 410 12; 14: 0 10 15; 15: 6 8 14; 16: 15 17; 17: 27 16; 18: 022 26; 19: 3034 38; 20: 22 25 27; 21: 23 25 26; 22: 18 20 23; 23: 2122 34; 24: 1125 42; 25: 2021 24; 26: 1821 27; 27: 2026 29; 28: 331 32; 29: 2736 39; 30: 1931 33; 31: 2830 42; 32: 2837 43; 33: 930 43; 34: 19 23 40; 35: 37 39 40; 36: 2937 41; 37: 3235 36; 38: 1939 41; 39: 29 35 38; 40: 3435 41; 41: 3638 40; 42: 2431 43; 43: 323342$\} \quad$ (Class 2)

28. $\{0: 12$ 3; 1: 040 41; 2: 039 43; 3: 038 42; 4: 9 13 17; 5: 812 17; 6: 7 15 19; 7: 616 18; 8: 59 14; 9: 48 11; 10: 13 16 22; 11: 912 20; 12: 511 26; 13: 410 25; 14: 8 15 21; 15: 6 
14 23; 16: 710 24; 17: 45 20; 18: 722 28; 19: 621 27; 20: 1117 32; 21: 14 19 37; 22: 10 18 36; 23: 1527 37; 24: 1628 36; 25: 13 30 35; 26: 12 29 34; 27: 19 23 32; 28: 1824 33; 29: 2631 35; 30: 2531 34; 31: 2930 33; 32: 2027 40; 33: 2831 41; 34: 2630 39; 35: 25 29 39; 36: 2224 38; 37: 2123 38; 38: 336 37; 39: 234 35; 40: 132 43; 41: 133 42; 42: 3 41 43; 43: 24042$\} \quad$ (Class 5a)

29. $\{0: 12$ 3; 1: 040 41; 2: 039 43; 3: 038 42; 4: 913 17; 5: 812 17; 6: 715 19; 7: 616 18; 8: 59 14; 9: 48 11; 10: 13 16 22; 11: 912 20; 12: 511 26; 13: 410 25; 14: 815 21; 15: 6 14 23; 16: 710 24; 17: 45 20; 18: 722 28; 19: 621 27; 20: 1117 32; 21: 14 19 37; 22: 10 18 36; 23 : 1527 37; 24: 1628 36; 25: 13 31 35; 26: 12 29 33; 27: 1923 32; 28 : 1824 34; 29: 2630 35; 30: 2931 34; 31: 2530 33; 32: 2027 40; 33: 2631 41; 34: 2830 39; 35: 25 29 39; 36: 2224 38; 37: 21 23 38; 38: 336 37; 39: 2 34 35; 40: 132 43; 41: 133 42; 42: 3 $4143 ; 43: 24042\} \quad$ (Class 6)

30. $\{0: 12$ 3; 1: 038 39; 2: 041 43; 3: 040 42; 4: 56 37; 5: 430 36; 6: 4 29 35; 7: 89 22; 8: 715 21; 9: 714 20; 10: 1418 31; 11: 1519 32; 12: 1417 34; 13: 1516 33; 14: 910 12; 15: 811 13; 16: 1326 32; 17: 1225 31; 18: 1025 34; 19: 1126 33; 20: 921 24; $21: 820$ 23; 22: 723 24; 23: 2122 28; 24: 2022 27; 25: 1718 28; 26: 1619 27; 27: 24 26 30; 28: 2325 29; 29: 628 36; 30: 527 35; 31: 1017 39; 32: 11 16 39; 33: 1319 43; 34 : 1218 42; 35: 630 38; 36: 529 38; 37: 440 41; 38: 135 36; 39: 131 32; 40: 3 37 43; 41: 237 42; 42: 334 41; 43: 233 40\} (Class 5b)

31. $\{0: 12$ 3; 1: 039 42; 2: 038 43; 3: 040 41; 4: 710 18; 5: 89 15; 6: 912 16; 7: 413 17; 8: 510 12; 9: 56 11; 10: 48 11; 11: 910 21; 12: 68 21; 13: 714 30; 14: 13 19 22; 15: 5 20 24; 16: 626 29; 17: 722 27; 18: 425 28; 19: 1420 23; 20: 1519 32; 21 : 11 12 35; 22: 1417 33; 23: 1931 35; 24: 1531 36; 25: 1829 37; 26: 1628 37; 27: 17 30 36; 28: 1826 34; 29: 16 25 34; 30: 1327 33; 31: 23 24 32; 32: 2031 38; 33: 22 30 39; 34: 28 29 39; 35: 2123 41; 36: 2427 38; 37: 25 26 40; 38: 2 32 36; 39: 133 34; 40: 337 43; 41: 335 42; 42: $14143 ; 43: 24042\} \quad$ (Class 6) 